TRANSACTIONS OF THE

AMERICAN MATHEMATICAL SOCIETY

Volume 361, Number 9, September 2009, Pages 4843-4870

S 0002-9947(09)04655-8

Article electronically published on April 17, 2009

\title{
ELLIPTIC EQUATIONS WITH CRITICAL GROWTH AND A LARGE SET OF BOUNDARY SINGULARITIES
}

\author{
NASSIF GHOUSSOUB AND FRÉDÉRIC ROBERT
}

\begin{abstract}
We solve variationally certain equations of stellar dynamics of the form $-\sum_{i} \partial_{i i} u(x)=\frac{|u|^{p-2} u(x)}{\operatorname{dist}(x, \mathcal{A})^{s}}$ in a domain $\Omega$ of $\mathbb{R}^{n}$, where $\mathcal{A}$ is a proper linear subspace of $\mathbb{R}^{n}$. Existence problems are related to the question of attainability of the best constant in the following inequality due to Maz'ya (1985):

$0<\mu_{s, \mathcal{P}}(\Omega)=\inf \left\{\int_{\Omega}|\nabla u|^{2} d x \mid u \in H_{1,0}^{2}(\Omega)\right.$ and $\left.\int_{\Omega} \frac{|u(x)|^{2^{\star}(s)}}{|\pi(x)|^{s}} d x=1\right\}$,

where $0<s<2,2^{\star}(s)=\frac{2(n-s)}{n-2}$ and where $\pi$ is the orthogonal projection on a linear space $\mathcal{P}$, where $\operatorname{dim}_{\mathbb{R}} \mathcal{P} \geq 2$ (see also Badiale-Tarantello (2002)). We investigate this question and how it depends on the relative position of the subspace $\mathcal{P}^{\perp}$, the orthogonal of $\mathcal{P}$, with respect to the domain $\Omega$, as well as on the curvature of the boundary $\partial \Omega$ at its points of intersection with $\mathcal{P}^{\perp}$.
\end{abstract}

\section{INTRODUCTION}

Let $\Omega$ be a smooth domain of $\mathbb{R}^{n}$, where $n \geq 3$, and denote by $H_{1,0}^{2}(\Omega)$ the completion of $C_{c}^{\infty}(\Omega)$, the set of smooth functions compactly supported in $\Omega$, for the norm $\|u\|_{H_{1,0}^{2}(\Omega)}=\sqrt{\int_{\Omega}|\nabla u|^{2} d x}$. In [20] (Corollary 2 in 2.1.6), Maz'ya proved that if $\mathcal{P}$ is a linear subspace of $\mathbb{R}^{n}$ such that $2 \leq \operatorname{dim}_{\mathbb{R}} \mathcal{P} \leq n$, then there exists $C>0$ such that for all $u \in H_{1,0}^{2}\left(\mathbb{R}^{n}\right)$,

$$
\left(\int_{\mathbb{R}^{n}} \frac{|u|^{2^{\star}}}{|\pi(x)|^{s}} d x\right)^{\frac{2}{2^{\star}}} \leq C \int_{\mathbb{R}^{n}}|\nabla u|^{2} d x
$$

where here $2^{\star}:=\frac{2(n-s)}{n-2}, s \in(0,2)$ and $\pi$ is the orthogonal projection on $\mathcal{P}$ with respect to the Euclidean structure. Recently, an alternative proof of this inequality was given by Badiale and Tarantello [1]. Define

$$
\mu_{s, \mathcal{P}}(\Omega):=\inf \left\{\frac{\int_{\Omega}|\nabla u|^{2} d x}{\left(\int_{\Omega} \frac{|u|^{\star}}{|\pi(x)|^{s}} d x\right)^{\frac{2}{2^{\star}}}} \mid u \in H_{1,0}^{2}(\Omega) \backslash\{0\}\right\}
$$

Received by the editors February 28, 2006 and, in revised form, October 2, 2007.

2000 Mathematics Subject Classification. Primary 35J35; Secondary 35B40.

This research was partially supported by the Natural Sciences and Engineering Research Council of Canada. The first author gratefully acknowledges the hospitality and support of the Université de Nice where this work was initiated.

The second author gratefully acknowledges the hospitality and support of the University of British Columbia. 
and note that (11) and (2) give that for all smooth domain $\Omega \subset \mathbb{R}^{n}$, we have

$$
\mu_{s, \mathcal{P}}(\Omega) \geq \mu_{s, \mathcal{P}}\left(\mathbb{R}^{n}\right)>0 .
$$

In this article, we adress the question of the value of the best constant $\mu_{s, \mathcal{P}}(\Omega)$ as well as the issue of its attainability. As we will see, both questions are closely related to the relative positions of $\mathcal{P}^{\perp}$ and $\Omega$, and to the geometry of the boundary $\partial \Omega$ at the points of $\mathcal{P}^{\perp} \cap \partial \Omega$.

The case when $s=0$ (i.e., the nonsingular case) is the well-known Sobolev inequality. In this situation the infimum $\mu_{s, \mathcal{P}}(\Omega)=\mu_{0, \mathcal{P}}\left(\mathbb{R}^{n}\right)$ is not attained unless $\Omega$ is essentially the whole of $\mathbb{R}^{n}$.

The case $s \in(0,2)$ and $\operatorname{dim}_{\mathbb{R}} \mathcal{P}=n$ (that is $\mathcal{P}=\mathbb{R}^{n}$ ) was tackled in [11, [12], 13. It was proved that when $0 \in \partial \Omega$, the infimum in (2) is then attained as soon as the mean curvature of $\partial \Omega$ (oriented with outward pointing normal vectors) at 0 is negative. The proof of this result required refined asymptotics for blown-up solutions of associated second order elliptic equations, the difficult case being when these solutions develop a "bubble" located precisely at the point 0 . However, the bubble inherits the symmetry properties of the problem, which allowed us to show in [12] that mean curvature conditions -as opposed to sectional curvature- suffice to eliminate the possibility of a bubbling-off phenomenon.

In the present paper, we tackle the case of a larger affine subspace of singularities $\left(1 \leq \operatorname{dim}_{\mathbb{R}} \mathcal{P} \leq n-1\right)$ and in particular when $\mathcal{P}^{\perp}$ contains at least a line. The situation here closely depends on the relative positions of $\mathcal{P}^{\perp}$ and $\Omega$, the most interesting case being when the subspace $\mathcal{P}^{\perp}$ does not touch the domain $\Omega$ but does touch its boundary (i.e., when $\mathcal{P}^{\perp} \cap \Omega=\emptyset$ and $\mathcal{P}^{\perp} \cap \partial \Omega \neq \emptyset$ ). A large part of the analysis is similar to what we have done in [12, 13, for the case of a single point of singularity on the boundary of $\Omega$. However, a new set of difficulties arises in this situation: for one, the centers of the appearing bubbles are not bound to any particular location and may appear anywhere on $\partial \Omega$. They do eventually converge to a point in $\mathcal{P}^{\perp} \cap \partial \Omega \neq \emptyset$, and an important new issue becomes the precise control of the distance between the center of the bubble and this limiting point.

Another new problem related to this setting is the lack of symmetry of the bubble. As described by the next proposition, we do show that it enjoys the best symmetry possible in the $\mathcal{P}$-direction. Here and in the sequel, $\Delta=-\sum_{i} \partial_{i i}$ will denote the Laplacian with minus sign convention and $\mathbb{R}_{-}^{n}=\left\{x \in \mathbb{R}_{-}^{n} / x_{1}<0\right\}$.

Proposition 1.1. Let $\pi$ be the projection on a linear subspace $\mathcal{Q}$ of $\mathbb{R}^{n}$ such that $2 \leq \operatorname{dim}_{\mathbb{R}} \mathcal{Q}$ and $\mathcal{Q}^{\perp} \subset \partial \mathbb{R}_{-}^{n}$. Assume $s \in(0,2)$ and consider $u \in C^{2}\left(\mathbb{R}_{-}^{n}\right) \cap C^{1}\left(\overline{\mathbb{R}_{-}^{n}}\right)$ such that

$$
\begin{cases}\Delta u=\frac{u^{2^{\star}-1}}{|\pi(x)|^{s}} & \text { in } \mathbb{R}_{-}^{n}, \\ u>0 & \text { in } \mathbb{R}_{-}^{n}, \\ u=0 & \text { on } \partial \mathbb{R}_{-}^{n},\end{cases}
$$

and for some $C>0$,

$$
u(x) \leq C(1+|x|)^{1-n} \text { for all } x \in \mathbb{R}_{-}^{n} .
$$

Then there exists $v \in C^{2}\left(\mathbb{R}_{-}^{\star} \times \mathbb{R} \times \mathcal{Q}^{\perp}\right) \cap C^{1}\left(\mathbb{R}_{-} \times \mathbb{R} \times \mathcal{Q}^{\perp}\right)$ such that for all $z \in \mathcal{Q}^{\perp}$, and all $x_{1}<0$ and $y \in \mathbb{R}^{n}$ with $\left(x_{1}, y\right) \in \mathcal{Q}$, we have that $u\left(x_{1}, y, z\right)=v\left(x_{1},|y|, z\right)$.

But this is not sufficient since the behavior of the bubble in the $\mathcal{P}$-direction and the $\mathcal{P}^{\perp}$-direction often cannot be related. Overcoming these difficulties, we prove 
the following theorem. In the sequel, $T_{x} \partial \Omega$ denotes the tangent space of $\partial \Omega$ at the point $x$.

Theorem 1.1. Let $\Omega$ be a smooth bounded oriented domain of $\mathbb{R}^{n}, n \geq 3$, and let $\mathcal{P}$ be a linear subspace of $\mathbb{R}^{n}$ such that $2 \leq \operatorname{dim}_{\mathbb{R}} \mathcal{P}$. Assume $s \in(0,2)$.

(A) If $\mathcal{P}^{\perp} \cap \Omega \neq \emptyset$, then $\mu_{s, \mathcal{P}}(\Omega)=\mu_{s, \mathcal{P}}\left(\mathbb{R}^{n}\right)$ and the infimum in (2) is not achieved.

(B) If $\mathcal{P}^{\perp} \cap \bar{\Omega}=\emptyset$, then the infimum in (2) is achieved.

(C) If $\mathcal{P}^{\perp} \cap \Omega=\emptyset$ and $\mathcal{P}^{\perp} \cap \partial \Omega \neq \emptyset$, then the infimum in (2) is achieved and the set of minimizers is pre-compact in $H_{1,0}^{2}(\Omega)$, provided that at any point $x \in \mathcal{P}^{\perp} \cap \partial \Omega$ the principal curvatures of $\partial \Omega$ at $x$ are nonpositive, but do not all vanish.

Moreover, at those points $x \in \mathcal{P}^{\perp} \cap \partial \Omega$ where $\mathcal{P} \cap T_{x} \partial \Omega$ and $\mathcal{P}^{\perp}$ are orthogonal with respect to the second fundamental form of $\partial \Omega$ at $x$, it suffices that the mean curvature vector of $\partial \Omega \cap\left(x+\left(\mathcal{P}^{\perp}+\left(T_{x} \partial \Omega\right)^{\perp}\right)\right)$ at $x$ be null, while the mean curvature of $\partial \Omega$ at $x$ is negative.

The second part in (C) makes a connection with the case where $\mathcal{P}=\mathbb{R}^{n}$ (i.e., $\mathcal{P}^{\perp}=\{0\}$ studied in [12]). Then the negativity of the mean curvature of $\partial \Omega$ at that point is sufficient for $\mu_{s, \mathcal{P}}(\Omega)$ to be attained. One may ask what happens in the case when $\operatorname{dim}_{\mathbb{R}} \mathcal{P} \in\{0,1\}$. In the case when $\mathcal{P}=\{0\}$, inequality (1) is clearly irrelevant. However the case $\operatorname{dim}_{\mathbb{R}} \mathcal{P}=1$ presents some interest, and this is the object of the following proposition:

Proposition 1.2. Let $\Omega$ be a smooth bounded oriented domain of $\mathbb{R}^{n}, n \geq 3$, and let $\mathcal{P}$ be a linear subspace of $\mathbb{R}^{n}$ such that $\operatorname{dim}_{\mathbb{R}} \mathcal{P}=1$. Assume $s \in(0,2)$.

(A) If $\mathcal{P}^{\perp} \cap \Omega \neq \emptyset$, then the infimum in (2) is not achieved.

(B) If $\mathcal{P}^{\perp} \cap \bar{\Omega}=\emptyset$, then the infimum $\mu_{s, \mathcal{P}}(\Omega)$ in (2) is positive and is achieved.

(C) If $\mathcal{P}^{\perp} \cap \Omega=\emptyset$ while $\mathcal{P}^{\perp} \cap \partial \Omega \neq \emptyset$, then $\mu_{s, \mathcal{P}}(\Omega)>0$ and the infimum is not achieved.

Actually, when dealing with case (C) of Theorem 1.1 and Proposition 1.2, the crucial point is to have negative principal curvatures at each point of $\mathcal{P}^{\perp} \cap \partial \Omega$. But the fact that $\mathcal{P}^{\perp}$ only touches $\bar{\Omega}$ at its boundary means that the principal curvatures in the $\mathcal{P}^{\perp}$-direction are all nonnegative at these points -at least for those where $\mathcal{P}^{\perp}$ and $\mathcal{P} \cap T_{x} \partial \Omega$ are orthogonal for the fundamental form of $\partial \Omega$. Therefore, for $\mu_{s, \mathcal{P}}(\Omega)$ to be achieved, one needs the negativity of the principal curvatures in some of the orthogonal directions, which is obviously impossible if $\mathcal{P}^{\perp}$ is $(n-1)$-dimensional, and therefore the best constant is never achieved in this case. This means that the dimension restriction on the linear subspace in Theorem 1.1 is optimal. As a consequence of the techniques developed for the proof of Theorem 1.1, we get the following corollary.

Corollary 1.1. Let $\Omega$ be a smooth bounded oriented domain of $\mathbb{R}^{n}$ and let $\pi$ be the orthogonal projection onto a linear subspace $\mathcal{Q} \subset \mathbb{R}^{n}$ such that $2 \leq \operatorname{dim}_{\mathbb{R}} \mathcal{Q}$. We assume that $\mathcal{Q}^{\perp} \cap \Omega=\emptyset$ and $\mathcal{Q}^{\perp} \cap \partial \Omega \neq \emptyset$. Assume $s \in(0,2)$ and consider $a \in C^{1}(\bar{\Omega})$ such that the operator $\Delta+a$ is coercive on $\Omega$. Then there exists a 
solution $u \in H_{1,0}^{2}(\Omega) \cap C^{1}(\bar{\Omega})$ for

$$
\begin{cases}\Delta u+a u=\frac{u^{2^{\star}-1}}{|\pi(x)|^{s}} & \text { in } \mathcal{D}^{\prime}(\Omega), \\ u>0 & \text { in } \Omega, \\ u=0 & \text { on } \partial \Omega,\end{cases}
$$

provided that at any point $x \in \mathcal{Q}^{\perp} \cap \partial \Omega$ the principal curvatures of $\partial \Omega$ at $x$ are nonpositive, but do not all vanish.

Moreover, at those points $x \in \partial \Omega \cap \mathcal{Q}^{\perp}$ where $\mathcal{Q}^{\perp}$ and $\mathcal{Q} \cap T_{x} \partial \Omega$ are orthogonal with respect to the second fundamental form of $\partial \Omega$ at $x$, it suffices to assume that the mean curvature vector of $\partial \Omega \cap\left(x+\left(\mathcal{Q}^{\perp}+\left(T_{x} \partial \Omega\right)^{\perp}\right)\right)$ at $x$ is null, while the mean curvature of $\partial \Omega$ at $x$ is negative.

Related references for best constant problems in Sobolev inequalities are Druet [5], Hebey-Vaugon [18, 19] and Egnell [10. Concerning asymptotics for blown-up sequences of solutions to elliptic equations, we also refer to Atkinson-Peletier 2], Brézis-Peletier [3], Han [17, Druet [6], Druet-Hebey [7, Druet-Hebey-Robert [8] and Schoen-Zhang [23].

The rest of the paper is devoted to the proof of these results. As mentioned above, a significant part of the analysis was developed in [12, 13] for the case of a unique singular point at the boundary, and to which we shall refer frequently. On the other hand, we shall give all the details relating to the new difficulties arising in this new setting of large sets of singularities. The paper is organized as follows. In section 2, we deal with points (A) and (B) of Theorem 1.1 and prove a symmetry result. Sections 3 to 5 are devoted to the proof of point (C) of Theorem 1.1 which is much more intricate, as it will require the full range of modern techniques for blow-up analysis and strong pointwise estimates for minimizers of the subcritical functional associated to (22). In section 6, we prove Proposition 1.2, while the appendix in section 7 provides a required regularity result for the family of elliptic pde's with singularities that we are dealing with in this paper. As a last remark, note that all the statements can be straightforwardly adapted to the case when $\mathcal{P}$ is an affine subspace of $\mathbb{R}^{n}$, and not only a linear space.

\section{Partial symmetry of bubbles and Parts (A), (B) of Theorem 1.1}

We let $\mathcal{P}$ be a linear subspace of $\mathbb{R}^{n}$ with $2 \leq \operatorname{dim}_{\mathbb{R}} \mathcal{P} \leq n-1$. We shall denote by $\pi$ the orthogonal projection on $\mathcal{P}$, and

$$
\mu_{s, \mathcal{P}}(\Omega):=\inf \left\{\frac{\int_{\Omega}|\nabla u|^{2} d x}{\left(\int_{\Omega} \frac{|u|^{\star}}{|\pi(x)|^{s}} d x\right)^{\frac{2}{2^{\star}}}} \mid u \in H_{1,0}^{2}(\Omega) \backslash\{0\}\right\} .
$$

Proof of Proposition 1.1. We first prove the partial symmetry property for the positive solutions to the limit equation on $\mathbb{R}_{-}^{n}$. For that, we consider $u \in C^{2}\left(\mathbb{R}_{-}^{n}\right) \cap$ $C^{1}\left(\overline{\mathbb{R}_{-}^{n}}\right)$ that verifies the system (4) while verifying for some $C>0$ the bound $u(x) \leq \frac{C}{(1+|x|)^{n-1}}$. We follow the proof of [12] to which we refer for details. For simplicity, up to a change of coordinates, we write any point $x \in \mathbb{R}^{n}$ as $x=\left(x_{1}, y, z\right)$, where $\left(x_{1}, y\right) \in \mathcal{Q}=\mathbb{R}^{k}$ and $z \in \mathcal{Q}^{\perp}=\mathbb{R}^{n-k}$. Therefore $\pi(x)=\left(x_{1}, y, 0\right)$. We let 
$\vec{e}_{1}$ be the first vector of the canonical basis of $\mathbb{R}^{n}$ and consider the open ball

$$
D:=B_{1 / 2}\left(-\frac{1}{2} \vec{e}_{1}\right) \text {. }
$$

We define

$$
v(x):=|x|^{2-n} u\left(\vec{e}_{1}+\frac{x}{|x|^{2}}\right)
$$

for all $x \in \bar{D} \backslash\{0\}$. We extend $v$ by 0 at 0 . This is then well-defined and $v \in$ $C^{2}(D) \cap C^{1}(\bar{D} \backslash\{0\})$. Moreover, $v(x)>0$ for all $x \in D$ and $v(x)=0$ for all $x \in \partial D \backslash\{0\}$. The function $v$ verifies the equation

$$
\Delta v=\frac{v^{2^{\star}-1}}{\left|\pi\left(x+|x|^{2} \vec{e}_{1}\right)\right|^{s}}
$$

in $D$. Since $v>0$ in $D$, it follows from Hopf's Lemma that $\frac{\partial v}{\partial \nu}<0$ on $\partial D \backslash\{0\}$.

We prove the symmetry of $u$ by proving a symmetry property of $v$, which is defined on a ball. Our proof uses the moving plane method. We largely take inspiration in 4 and [15. We let $i \in\{2, \ldots, k\}$. For any $\mu \geq 0$ and $x \in \mathbb{R}^{n}$, we let

$$
x_{\mu}=\left(x_{1}, \ldots, 2 \mu-x_{i}, \ldots, x_{n}\right) \text { and } D_{\mu}=\left\{x \in D / x_{\mu} \in D\right\} .
$$

It follows from Hopf's Lemma that there exists $\epsilon_{0}>0$ such that for any $\mu \in$ $\left(\frac{1}{2}-\epsilon_{0}, \frac{1}{2}\right)$, we have that $D_{\mu} \neq \emptyset$ and $v(x) \geq v\left(x_{\mu}\right)$ for all $x \in D_{\mu}$ such that $x_{i} \leq \mu$. We let $\mu \geq 0$. We say that $\left(P_{\mu}\right)$ holds if $D_{\mu} \neq \emptyset$ and $v(x) \geq v\left(x_{\mu}\right)$ for all $x \in D_{\mu}$ such that $x_{i} \leq \mu$. We let

$$
\lambda:=\min \left\{\mu \geq 0 \mid\left(P_{\nu}\right) \text { holds for all } \nu \in\left(\mu, \frac{1}{2}\right)\right\} .
$$

We claim that $\lambda=0$. Indeed we proceed by contradiction and assume that $\lambda>0$. We then get that $D_{\lambda} \neq \emptyset$ and that $\left(P_{\lambda}\right)$ holds. We let $w(x):=v(x)-v\left(x_{\lambda}\right)$ for all $x \in D_{\lambda} \cap\left\{x_{n}<\lambda\right\}$. Since $\left(P_{\lambda}\right)$ holds, we have that $w(x) \geq 0$ for all $x \in D_{\lambda} \cap\left\{x_{i}<\lambda\right\}$. With equation (8) of $v$ and $\left(P_{\lambda}\right)$, we get that

$$
\begin{aligned}
\Delta w & =\frac{v(x)^{2^{\star}-1}}{\left|\pi\left(x+|x|^{2} \vec{e}_{1}\right)\right|^{s}}-\frac{v\left(x_{\lambda}\right)^{2^{\star}-1}}{\left|\pi\left(x_{\lambda}+\left|x_{\lambda}\right|^{2} \vec{e}_{1}\right)\right|^{s}} \\
& \geq v\left(x_{\lambda}\right)^{2^{\star}-1}\left(\frac{1}{\left|\pi\left(x+|x|^{2} \vec{e}_{1}\right)\right|^{s}}-\frac{1}{\left|\pi\left(x_{\lambda}+\left|x_{\lambda}\right|^{2} \vec{e}_{1}\right)\right|^{s}}\right)
\end{aligned}
$$

for all $x \in D_{\lambda} \cap\left\{x_{i}<\lambda\right\}$. Since $2 \leq i \leq k$, we get that the RHS is positive (see [12]), and then $\Delta w(x)>0$ for all $x \in D_{\lambda} \cap\left\{x_{i}<\lambda\right\}$. It then follows from Hopf's Lemma and the strong comparison principle that

$$
w>0 \text { in } D_{\lambda} \cap\left\{x_{i}<\lambda\right\} \text { and } \frac{\partial w}{\partial \nu}<0 \text { on } D_{\lambda} \cap\left\{x_{i}=\lambda\right\} .
$$

The contradiction then follows from standard arguments; we refer to [12, 13] for details. This yields $\lambda=0$.

Here is the final argument. Since $\lambda=0$, it follows from the definition (9) of $\lambda$ that $v(x) \geq v\left(x_{1}, \ldots,-x_{i}, \ldots, x_{n}\right)$ for all $x \in D$ such that $x_{i} \leq 0$. With the same technique we get the reverse inequality, and then we get that $v(x)=$ $v\left(x_{1}, \ldots,-x_{i}, \ldots, x_{n}\right)$ for all $x=\left(x^{\prime}, x_{n}\right) \in D$. In other words, $v$ is symmetric with respect to the hyperplane $\left\{x_{i}=0\right\}$. The same analysis holds for any hyperplane 
containing $\operatorname{Span}\left\{\overrightarrow{e_{1}}, \overrightarrow{e_{k+1}}, \ldots, \overrightarrow{e_{n}}\right\}$. Coming back to the initial function $u$, this proves Proposition 1.1 and the symmetry property.

The object of the following proposition is to deal with case (A) of Theorem 1.1. that is, when $\mathcal{P}^{\perp} \cap \Omega \neq \emptyset$.

Proposition 2.1. Let $\Omega$ be a smooth bounded domain of $\mathbb{R}^{n}, n \geq 3$. Let $\mathcal{P} \subset \mathbb{R}^{n}$ be a linear subspace of $\mathbb{R}^{n}$, where $2 \leq \operatorname{dim}_{\mathbb{R}} \mathcal{P} \leq n-1$. Let $s \in(0,2)$ and assume that $\mathcal{P}^{\perp} \cap \Omega \neq \emptyset$. Then $\mu_{s, \mathcal{P}}(\Omega)=\mu_{s, \mathcal{P}}\left(\mathbb{R}^{n}\right)$, and the infimum $\mu_{s, \mathcal{P}}(\Omega)$ is not achieved.

Proof. Fix $x_{0} \in \mathcal{P}^{\perp} \cap \Omega$, and let $\delta>0$ such that $B_{\delta}\left(x_{0}\right) \subset \Omega$. Let $\alpha>0$ and $u \in C_{c}^{\infty}\left(\mathbb{R}^{n}\right) \backslash\{0\}$ such that

$$
\frac{\int_{\mathbb{R}^{n}}|\nabla u|^{2} d x}{\left(\int_{\mathbb{R}^{n}} \frac{|u|^{2^{\star}}}{|\pi(x)|^{s}} d x\right)^{\frac{2}{2^{\star}}}} \leq \mu_{s, \mathcal{P}}\left(\mathbb{R}^{n}\right)+\alpha .
$$

For $\epsilon>0$, we let $u_{\epsilon}(x):=\epsilon^{-\frac{n-2}{2}} u\left(\frac{x-x_{0}}{\epsilon}\right)$ for all $x \in \Omega$. As is easily checked, $u_{\epsilon} \in C_{c}^{\infty}(\Omega)$ for $\epsilon>0$ small and

$$
\frac{\int_{\Omega}\left|\nabla u_{\epsilon}\right|^{2} d x}{\left(\int_{\Omega} \frac{\left|u_{\epsilon}\right|^{\star}}{|\pi(x)|^{s}} d x\right)^{\frac{2}{2^{\star}}}}=\frac{\int_{\mathbb{R}^{n}}|\nabla u|^{2} d x}{\left(\int_{\mathbb{R}^{n}} \frac{|u|^{2^{\star}}}{|\pi(x)|^{s}} d x\right)^{\frac{2}{2^{\star}}}} \leq \mu_{s, \mathcal{P}}\left(\mathbb{R}^{n}\right)+\alpha .
$$

Here, we have used the fact that $x_{0} \in \mathcal{P}^{\perp}$; that is, $\pi\left(x_{0}\right)=0$. Coming back to the definition (6) of $\mu_{s, \mathcal{P}}(\Omega)$ letting $\alpha \rightarrow 0$ and using (3), we get that $\mu_{s, \mathcal{P}}(\Omega)=$ $\mu_{s, \mathcal{P}}\left(\mathbb{R}^{n}\right)$.

We claim that $\mu_{s, \mathcal{P}}(\Omega)$ is not achieved. Indeed, assuming it is achieved by a function $u \in H_{1,0}^{2}(\Omega) \backslash\{0\}$, we can assume without loss that $u \geq 0$. Since $\mu_{s, \mathcal{P}}(\Omega)=\mu_{s, \mathcal{P}}\left(\mathbb{R}^{n}\right)$, we get that $\mu_{s, \mathcal{P}}\left(\mathbb{R}^{n}\right)$ is also attained by $u$, which then verifies $\Delta u=\frac{u^{2^{\star}-1}}{|\pi(x)|^{s}}$ in $\mathcal{D}^{\prime}\left(\mathbb{R}^{n}\right)$. Since $u \geq 0$, it follows from the regularity results of section 7 and the maximum principle that $u>0$ on $\mathbb{R}^{n} \backslash \mathcal{P}$, a contradiction since $u \in H_{1,0}^{2}(\Omega)$.

The case where $\mathcal{P}^{\perp} \cap \bar{\Omega}=\emptyset$ is dealt with in the following proposition.

Proposition 2.2. Let $\Omega$ be a smooth bounded domain of $\mathbb{R}^{n}, n \geq 3$, and let $\mathcal{P}$ be a linear subspace of $\mathbb{R}^{n}$ such that $2 \leq \operatorname{dim}_{\mathbb{R}} \mathcal{P} \leq n-1$. Assume $s \in(0,2)$ and that $\mathcal{P}^{\perp} \cap \bar{\Omega}=\emptyset$; then the infimum $\mu_{s, \mathcal{P}}(\Omega)$ is attained.

Proof. Since $\mathcal{P}^{\perp} \cap \bar{\Omega}=\emptyset$, there exists $c, C>0$ such that $c \leq|\pi(x)| \leq C$ for all $x \in \Omega$. In particular, since $2^{\star}<\frac{2 n}{n-2}$, we have compactness of the embedding of $H_{1,0}^{2}(\Omega)$ in $L^{2^{\star}}\left(\Omega,|\pi(x)|^{-s}\right)$ and therefore the existence of minimizers. This ends the proof of the proposition.

\section{Blow-up analysis, Part I}

Throughout this section, we let $\Omega$ be a smooth bounded domain of $\mathbb{R}^{n}, n \geq 3$, and let $\mathcal{P}$ be a linear subspace of $\mathbb{R}^{n}$ such that $2 \leq \operatorname{dim}_{\mathbb{R}} \mathcal{P} \leq n-1$. Let $s \in(0,2)$ and assume that

$$
\mathcal{P}^{\perp} \cap \Omega=\emptyset \text { and } \mathcal{P}^{\perp} \cap \partial \Omega \neq \emptyset .
$$

Here and in the sequel, we let $\pi$ be the orthogonal projection on $\mathcal{P}$. This is the most intricate case to which the rest of the paper is essentially devoted. 
Proposition 3.1. Let $\Omega$ be a smooth bounded domain of $\mathbb{R}^{n}, n \geq 3$, and let $\mathcal{P}$ be a linear subspace of $\mathbb{R}^{n}$, such that $2 \leq \operatorname{dim}_{\mathbb{R}} \mathcal{P} \leq n-1$. Let $s \in(0,2)$ and assume that $\mathcal{P}^{\perp} \cap \Omega=\emptyset$ and $\mathcal{P}^{\perp} \cap \partial \Omega \neq \emptyset$; then $\mu_{s, \mathcal{P}}(\Omega) \leq \mu_{s, \mathcal{P}}\left(\mathbb{R}_{-}^{n}\right)$.

Proof. Let $x_{0} \in \mathcal{P}^{\perp} \cap \partial \Omega$. Since $\mathcal{P}^{\perp} \cap \Omega=\emptyset$, we have that

$$
\mathcal{P}^{\perp} \subset T_{x_{0}} \partial \Omega
$$

where $T_{x_{0}} \partial \Omega$ is the tangent space at $x_{0}$ of the smooth manifold $\partial \Omega$. It follows from (11) that $\left(T_{x_{0}} \partial \Omega\right)^{\perp} \subset \mathcal{P}$. We choose a direct orthonormal basis $\left(\vec{e}_{1}, \ldots, \vec{e}_{n}\right)$ of $\mathbb{R}^{n}$ such that

$$
\begin{aligned}
& \vec{e}_{1}=\vec{n}_{x_{0}} \text { is the normal outward vector at } x_{0} \text { of } \partial \Omega, \\
& \left(\vec{e}_{1}, \ldots, \vec{e}_{k}\right) \text { is an orthonormal basis of } \mathcal{P} \\
& \left(\vec{e}_{k+1}, \ldots, \vec{e}_{n}\right) \text { is an orthonormal basis of } \mathcal{P}^{\perp} .
\end{aligned}
$$

Here and in what follows, $k=\operatorname{dim}_{\mathbb{R}} \mathcal{P}$, so that $2 \leq k \leq n-1$. In particular, $\left(\vec{e}_{2}, \ldots, \vec{e}_{n}\right)$ is an orthonormal basis of $T_{x_{0}} \partial \Omega$. For the rest of this section, we shall be referring to this particular basis. In particular, we adopt the following notation: we write any element $x \in \mathbb{R}^{n}$ as $x=\left(x_{1}, y, z\right)$, with $x_{1} \in \mathbb{R}, y \in \operatorname{span}\left(\vec{e}_{2}, \ldots, \vec{e}_{k}\right)$ and $z \in \operatorname{span}\left(\vec{e}_{k+1}, \ldots, \vec{e}_{n}\right)=\mathcal{P}^{\perp}$.

Since $\partial \Omega$ is smooth, there exist $U, V$ open subsets of $\mathbb{R}^{n}$, such that $0 \in U$ and $x_{0} \in V$, and there exists $\varphi \in C^{\infty}(U, V)$ and $\varphi_{0} \in C^{\infty}\left(U^{\prime}\right)$ (where $U^{\prime}=$ $\left\{(y, z) / \exists x_{1} \in \mathbb{R}\right.$ s.t. $\left.\left.\left(x_{1}, y, z\right) \in U\right\}\right)$ such that

(i) $\varphi: U \rightarrow V$ is a $C^{\infty}-$ diffeomorphism,

(ii) $\varphi(0)=x_{0}$

(iii) $\varphi\left(U \cap\left\{x_{1}<0\right\}\right)=\varphi(U) \cap \Omega$ and $\varphi\left(U \cap\left\{x_{1}=0\right\}\right)=\varphi(U) \cap \partial \Omega$,

(iv) $\varphi_{0}(0)=0$ and $\nabla \varphi_{0}(0)=0$,

(v) $\varphi\left(x_{1}, y, z\right)=\left(x_{1}+\varphi_{0}(y, z), y, z\right)+x_{0}$ for all $\left(x_{1}, y, z\right) \in U$,

where $D_{x} \varphi_{0}$ denotes the differential of $\varphi_{0}$ at $x$. Let $\alpha>0$ and $u \in C_{c}^{\infty}\left(\mathbb{R}_{-}^{n}\right) \backslash\{0\}$ such that

$$
\frac{\int_{\mathbb{R}_{-}^{n}}|\nabla u|^{2} d x}{\left(\int_{\mathbb{R}_{-}^{n}} \frac{|u|^{\star}}{|\pi(x)|^{s}} d x\right)^{\frac{2}{2^{\star}}}} \leq \mu_{s, \mathcal{P}}\left(\mathbb{R}_{-}^{n}\right)+\alpha
$$

Define $u_{\epsilon}(x):=\epsilon^{-\frac{n-2}{2}} u\left(\frac{\varphi^{-1}(x)}{\epsilon}\right)$ for all $x \in \Omega$ and all $\epsilon>0$. As is easily checked, for $\epsilon>0$ small enough, we have that $u_{\epsilon} \in C_{c}^{\infty}(\Omega)$. Standard computations yield that

$$
\mu_{s, \mathcal{P}}(\Omega) \leq \frac{\int_{\Omega}\left|\nabla u_{\epsilon}\right|^{2} d x}{\left(\int_{\Omega} \frac{\left|u_{\epsilon}\right|^{\star}}{|\pi(x)|^{s}} d x\right)^{\frac{2}{2^{\star}}}}=\frac{\int_{\mathbb{R}_{-}^{n}}|\nabla u|^{2} d x}{\left(\int_{\mathbb{R}_{-}^{n}} \frac{|u|^{2^{\star}}}{|\pi(x)|^{s}} d x\right)^{\frac{2}{2^{\star}}}}+o(1) \leq \mu_{s, \mathcal{P}}\left(\mathbb{R}_{-}^{n}\right)+\alpha+o(1),
$$

where $\lim _{\epsilon \rightarrow 0} o(1)=0$. Letting $\epsilon \rightarrow 0$ and $\alpha \rightarrow 0$, we get the claimed result.

In order to construct minimizers for $\mu_{s, \mathcal{P}}(\Omega)$, we consider a subcritical minimization problem for which we have compactness. The proof of this result is standard, and we refer to 12 for details.

Proposition 3.2. Let $\Omega$ be a smooth bounded domain of $\mathbb{R}^{n}, n \geq 3$, and let $\mathcal{P}$ be a linear subspace of $\mathbb{R}^{n}$ such that $2 \leq \operatorname{dim}_{\mathbb{R}} \mathcal{P} \leq n-1$. Let $s \in(0,2)$ and assume 
that (10) holds. Then for any $\epsilon \in\left(0,2^{\star}-2\right)$, the infimum

$$
\mu_{s, \mathcal{P}}^{\epsilon}(\Omega):=\inf _{u \in H_{1,0}^{2}(\Omega) \backslash\{0\}} \frac{\int_{\Omega}|\nabla u|^{2} d x}{\left(\int_{\Omega} \frac{|u|^{2^{\star}-\epsilon}}{|\pi(x)|^{s}} d x\right)^{\frac{2}{2^{\star}-\epsilon}}}
$$

is achieved by a function $u_{\epsilon} \in H_{1,0}^{2}(\Omega)$. Moreover, $u_{\epsilon} \in C^{\infty}\left(\bar{\Omega} \backslash \mathcal{P}^{\perp}\right)$ and can be assumed to satisfy the system

$$
\begin{cases}\Delta u_{\epsilon}=\frac{u_{\epsilon}^{2^{\star}-1-\epsilon}}{|\pi(x)|^{s}} & \text { in } \mathcal{D}^{\prime}(\Omega), \\ u_{\epsilon}>0 & \text { in } \Omega, \\ \int_{\Omega} \frac{u_{\epsilon}^{2^{\star}-\epsilon}}{|\pi(x)|^{s}} d x=\left(\mu_{s, \mathcal{P}}^{\epsilon}(\Omega)\right)^{\frac{2^{\star}-\epsilon}{2^{\star^{\star}-2-\epsilon}} .} & \end{cases}
$$

Moreover, we have that $\lim _{\epsilon \rightarrow 0} \mu_{s, \mathcal{P}}^{\epsilon}(\Omega)=\mu_{s, \mathcal{P}}(\Omega)$, and there exists $u_{0} \in H_{1,0}^{2}(\Omega)$ such that, up to a subsequence, $u_{\epsilon} \rightarrow u_{0}$ weakly in $H_{1,0}^{2}(\Omega)$ when $\epsilon \rightarrow 0$. If $u_{0} \not \equiv 0$, then $\lim _{\epsilon \rightarrow 0} u_{\epsilon}=u_{0}$ strongly in $H_{1,0}^{2}(\Omega)$ and $u_{0}$ is a minimizer for $\mu_{s, \mathcal{P}}(\Omega)$. In particular, $\mu_{s, \mathcal{P}}(\Omega)$ is attained.

We now start the blow-up analysis for minimizing sequences. Actually, we consider a more general case. Here and in the sequel, we let $p_{\epsilon} \in\left[0,2^{\star}-2\right)$ such that

$$
\lim _{\epsilon \rightarrow 0} p_{\epsilon}=0 .
$$

We assume that (10) holds. We consider a family $\left(a_{\epsilon}\right)_{\epsilon>0} \in C^{1}(\bar{\Omega})$ such that there exists $\lambda, C>0$ such that

$$
\left\|a_{\epsilon}\right\|_{C^{1}(\bar{\Omega})} \leq C \text { and } \int_{\Omega}\left(|\nabla \varphi|^{2}+a_{\epsilon} \varphi^{2}\right) d x \geq \lambda \int_{\Omega} \varphi^{2} d x
$$

for all $\epsilon \rightarrow 0$ and all $\varphi \in C_{c}^{\infty}(\Omega)$. For any $\epsilon>0$, we consider

$$
u_{\epsilon} \in H_{1,0}^{2}(\Omega) \cap C^{2}\left(\bar{\Omega} \backslash \mathcal{P}^{\perp}\right)
$$

a solution to the system

$$
\begin{cases}\Delta u_{\epsilon}+a_{\epsilon} u_{\epsilon}=\frac{u_{\epsilon}^{2^{\star}-1-p_{\epsilon}}}{|\pi(x)|^{s}} & \text { in } \mathcal{D}^{\prime}(\Omega), \\ u_{\epsilon}>0 & \text { in } \Omega .\end{cases}
$$

We assume that $u_{\epsilon}$ is of minimal energy type; that is,

$$
\int_{\Omega} \frac{\left|u_{\epsilon}\right|^{2^{\star}-p_{\epsilon}}}{|\pi(x)|^{s}} d x=\mu_{s, \mathcal{P}}(\Omega)^{\frac{2^{\star}}{2^{\star}-2}}+o(1),
$$

where $\lim _{\epsilon \rightarrow 0} o(1)=0$. We also assume that blow-up occurs; that is,

$$
u_{\epsilon} \rightarrow 0
$$

weakly in $H_{1,0}^{2}(\Omega)$ when $\epsilon \rightarrow 0$. Such a family arises naturally when $u_{0} \equiv 0$ in Proposition 3.2 It follows from Proposition 7.1 of the Appendix that $u_{\epsilon} \in C^{0}(\bar{\Omega})$. We let $x_{\epsilon} \in \Omega$ and $\mu_{\epsilon}, k_{\epsilon}>0$ such that

$$
\max _{\Omega} u_{\epsilon}=u_{\epsilon}\left(x_{\epsilon}\right)=\mu_{\epsilon}^{-\frac{n-2}{2}} \text { and } k_{\epsilon}:=\mu_{\epsilon}^{1-\frac{p_{\epsilon}}{2^{\star}-2}} .
$$


Our goal in this section is to prove the following:

Proposition 3.3. Under the above assumption, there exists $x_{0} \in \mathcal{P}^{\perp} \cap \partial \Omega$, a chart $\varphi$ as in (13), and there exists $\left(\bar{z}_{\epsilon}\right)_{\epsilon>0} \in \partial \mathbb{R}_{-}^{n}$ such that $\lim _{\epsilon \rightarrow 0} \bar{z}_{\epsilon}=0$ and such that the function

$$
v_{\epsilon}(x):=\mu_{\epsilon}^{\frac{n-2}{2}} u_{\epsilon} \circ \varphi\left(\bar{z}_{\epsilon}+k_{\epsilon} x\right)
$$

defined for $x \in \frac{U-\bar{z}_{\epsilon}}{k_{\epsilon}}$ and $\epsilon>0$ verifies that there exists $v \in H_{1,0}^{2}\left(\mathbb{R}_{-}^{n}\right) \backslash\{0\}$ such that for any $\eta \in C_{c}^{\infty}\left(\mathbb{R}^{n}\right), \eta v_{\epsilon} \rightarrow \eta v$ in $H_{1,0}^{2}\left(\mathbb{R}_{-}^{n}\right)$ weakly in $\mathcal{D}^{\prime}\left(\mathbb{R}_{-}^{n}\right)$ when $\epsilon \rightarrow 0$. The function $v$ verifies that

$$
\Delta v=\frac{v^{2^{\star}-1}}{|\pi(x)|^{s}} \text { in } \mathcal{D}^{\prime}\left(\mathbb{R}_{-}^{n}\right)
$$

and $\int_{\mathbb{R}_{-}^{n}}|\nabla v|^{2} d x=\mu_{s, \mathcal{P}}(\Omega)^{\frac{2^{\star}}{2^{\star}-2}}=\mu_{s, \mathcal{P}}\left(\mathbb{R}_{-}^{n}\right)^{\frac{2^{\star}}{2^{\star-2}}}$. In addition, $v \in C^{1}\left(\overline{\mathbb{R}_{-}^{n}}\right)$ and

$$
\lim _{\epsilon \rightarrow 0} v_{\epsilon}=v \text { in } C_{l o c}^{1}\left(\overline{\mathbb{R}_{-}^{n}}\right) .
$$

Moreover,

$$
\lim _{\epsilon \rightarrow 0} \mu_{\epsilon}^{p_{\epsilon}}=1 .
$$

Proof. The proof goes in six steps.

Step 3.1. We claim that

$$
\mu_{\epsilon}=o(1) \text { and } \pi\left(x_{\epsilon}\right)=O\left(k_{\epsilon}\right)
$$

when $\epsilon \rightarrow 0$.

Indeed assume that $\lim _{\epsilon \rightarrow 0} \mu_{\epsilon} \neq 0$. Then up to a subsequence, there exists $C>0$ such that $\left|u_{\epsilon}(x)\right| \leq C$ for all $x \in \Omega$ and all $\epsilon>0$. Mimicking the proof of the Appendix, we obtain that there exists $C>0$ such that $\left\|u_{\epsilon}\right\|_{C^{1}(\bar{\Omega})} \leq C$. Since (17) holds, it follows from Ascoli's theorem that, up to a subsequence, $\lim _{\epsilon \rightarrow 0} u_{\epsilon}=0$ in $C^{0}(\bar{\Omega})$ - a contradiction with (16). This proves that $\lim _{\epsilon \rightarrow 0} \mu_{\epsilon}=0$.

To prove the second part of the claim assume that

$$
\lim _{\epsilon \rightarrow 0} \frac{\left|\pi\left(x_{\epsilon}\right)\right|}{k_{\epsilon}}=+\infty
$$

For any $\epsilon>0$, set

$$
\beta_{\epsilon}=\left|\pi\left(x_{\epsilon}\right)\right|^{\frac{s}{2}} u_{\epsilon}\left(x_{\epsilon}\right)^{\frac{2+p_{\epsilon}-2^{\star}}{2}}=\left|\pi\left(x_{\epsilon}\right)\right|^{\frac{s}{2}} k_{\epsilon}^{\frac{2-s}{2}} .
$$

It follows from the definition (22) of $\beta_{\epsilon}$ and (21) that

$$
\lim _{\epsilon \rightarrow 0} \beta_{\epsilon}=0, \lim _{\epsilon \rightarrow 0}\left(\frac{\beta_{\epsilon}}{k_{\epsilon}}\right)^{2}=+\infty \text { and } \lim _{\epsilon \rightarrow 0}\left(\frac{\beta_{\epsilon}}{\left|\pi\left(x_{\epsilon}\right)\right|}\right)^{2}=0
$$

when $\epsilon \rightarrow 0$.

Case 3.1.1. Assume first that there exists $\rho>0$ such that $\frac{d\left(x_{\epsilon}, \partial \Omega\right)}{\beta_{\epsilon}} \geq 2 \rho$ for all $\epsilon>0$. For $x \in B_{2 \rho}(0)$ and $\epsilon>0$, define

$$
v_{\epsilon}(x):=\frac{u_{\epsilon}\left(x_{\epsilon}+\beta_{\epsilon} x\right)}{u_{\epsilon}\left(x_{\epsilon}\right)} .
$$


This is well defined since $x_{\epsilon}+\beta_{\epsilon} x \in \Omega$ for all $x \in B_{2 \rho}(0)$. As is easily checked, with (15), we have that

$$
\Delta v_{\epsilon}+k_{\epsilon}^{2} a_{\epsilon}\left(x_{\epsilon}+\beta_{\epsilon} x\right) v_{\epsilon}=\frac{v_{\epsilon}^{2^{\star}-1-p_{\epsilon}}}{\left|\frac{\pi\left(x_{\epsilon}\right)}{\left|\pi\left(x_{\epsilon}\right)\right|}+\frac{\beta_{\epsilon}}{\left|\pi\left(x_{\epsilon}\right)\right|} \pi(x)\right|^{s}}
$$

weakly in $B_{2 \rho}(0)$. Since $0 \leq v_{\epsilon}(x) \leq v_{\epsilon}(0)=1$ for all $x \in B_{\rho}(0)$, it follows from standard elliptic theory and (23) that there exists $v \in C^{1}\left(B_{2 \rho}(0)\right)$ such that $v_{\epsilon} \rightarrow v$ in $C_{l o c}^{1}\left(B_{2 \rho}(0)\right)$ as $\epsilon \rightarrow 0$. In particular,

$$
v(0)=\lim _{\epsilon \rightarrow 0} v_{\epsilon}(0)=1 .
$$

With a change of variable and the definition (22) of $\beta_{\epsilon}$, we get that

$$
\begin{aligned}
& \int_{\Omega \cap B_{\rho \beta_{\epsilon}}\left(x_{\epsilon}\right)} \frac{u_{\epsilon}^{2^{\star}-p_{\epsilon}}}{|\pi(x)|^{s}} d x=\frac{u_{\epsilon}\left(x_{\epsilon}\right)^{2^{\star}-p_{\epsilon}} \beta_{\epsilon}^{n}}{\left|\pi\left(x_{\epsilon}\right)\right|^{s}} \int_{B_{\rho}(0)} \frac{v_{\epsilon}^{2^{\star}-p_{\epsilon}}}{\left|\frac{\pi\left(x_{\epsilon}\right)}{\left|\pi\left(x_{\epsilon}\right)\right|}+\frac{\beta_{\epsilon}}{\left|\pi\left(x_{\epsilon}\right)\right|} \pi(x)\right|^{s}} d x \\
& \geq\left(\frac{\beta_{\epsilon}}{k_{\epsilon}}\right)^{n-2} \mu_{\epsilon}^{-p_{\epsilon} \frac{n-2}{2^{\star}-2}} \int_{B_{\rho}(0)} \frac{v_{\epsilon}^{2^{\star}-p_{\epsilon}}}{\left|\frac{\pi\left(x_{\epsilon}\right)}{\left|\pi\left(x_{\epsilon}\right)\right|}+\frac{\beta_{\epsilon}}{\left|\pi\left(x_{\epsilon}\right)\right|} \pi(x)\right|^{s}} d x .
\end{aligned}
$$

Using (16), (23) and passing to the limit $\epsilon \rightarrow 0$ (note that $\mu_{\epsilon}^{-1} \geq 1$ for $\epsilon>0$ small), we get that $\int_{B_{\rho}(0)} v^{2^{\star}} d x=0$, and then $v \equiv 0$. This contradicts (24) and therefore (21) does not hold, which proves the claim in Case 3.1.1.

Case 3.1.2. Now assume that, up to a subsequence, $\lim _{\epsilon \rightarrow 0} \frac{d\left(x_{\epsilon}, \partial \Omega\right)}{\beta_{\epsilon}}=0$. We then get a contradiction by a rescaling of $u_{\epsilon}$ as in 12 . The proof uses the techniques of Case 3,1.1 and is rather similar to [12, to which we refer for the details.

In both cases, we have obtained a contradiction and Step 3.1 is established.

Step 3.2. Up to a subsequence, we claim that $x_{0}$ defined as

$$
x_{0}:=\lim _{\epsilon \rightarrow 0} x_{\epsilon}
$$

belongs to $\mathcal{P}^{\perp} \cap \partial \Omega$.

Indeed, it follows from (20) and (18) that $\pi\left(x_{0}\right)=0$; that is, $x_{0} \in \mathcal{P}^{\perp}$. Since $x_{0} \in \bar{\Omega}$, it follows from (10) that $x_{0} \in \mathcal{P}^{\perp} \cap \partial \Omega$.

Since (10) holds, we have that (11) holds. We choose a basis as in (12), and we choose a chart $\varphi$ as in (13). In particular, here again we let $k=\operatorname{dim}_{\mathbb{R}} \mathcal{P} \in$ $\{2, \ldots, n-1\}$.

\section{Step 3.3. Setting}

$$
x_{\epsilon}=\varphi\left(x_{1, \epsilon}, y_{\epsilon}, z_{\epsilon}\right),
$$

where $x_{1, \epsilon}<0, y_{\epsilon} \in \operatorname{span}\left(\vec{e}_{2}, \ldots, \vec{e}_{k}\right)$ and $z_{\epsilon} \in \operatorname{span}\left(\vec{e}_{k+1}, \ldots, \vec{e}_{n}\right)=\mathcal{P}^{\perp}$, we claim that

$$
d\left(x_{\epsilon}, \partial \Omega\right)=(1+o(1))\left|x_{1, \epsilon}\right|=O\left(k_{\epsilon}\right), y_{\epsilon}=O\left(k_{\epsilon}\right) \text { and } \varphi_{0}\left(0, z_{\epsilon}\right)=O\left(k_{\epsilon}\right)
$$

when $\epsilon \rightarrow 0$. Here $\varphi_{0}$ is as in (13). 
Proof of the claim. Our first remark is that

$$
d\left(x_{\epsilon}, \partial \Omega\right)=O\left(k_{\epsilon}\right)
$$

when $\epsilon \rightarrow 0$. Indeed, since $\mathcal{P}^{\perp} \cap \Omega=\emptyset$, we have that $x_{\epsilon}-\pi\left(x_{\epsilon}\right) \in \mathcal{P}^{\perp} \in \mathbb{R}^{n} \backslash \Omega$. Since $x_{\epsilon} \in \Omega$, there exists $t_{\epsilon} \in(0,1)$ such that $t_{\epsilon} x_{\epsilon}+\left(1-t_{\epsilon}\right) \cdot\left(x_{\epsilon}-\pi\left(x_{\epsilon}\right)\right) \in \partial \Omega$. Consequently,

$d\left(x_{\epsilon}, \partial \Omega\right) \leq\left|x_{\epsilon}-\left(t_{\epsilon} x_{\epsilon}+\left(1-t_{\epsilon}\right) \cdot\left(x_{\epsilon}-\pi\left(x_{\epsilon}\right)\right)\right)\right|=\left(1-t_{\epsilon}\right)\left|\pi\left(x_{\epsilon}\right)\right| \leq\left|\pi\left(x_{\epsilon}\right)\right|=O\left(k_{\epsilon}\right)$ when $\epsilon \rightarrow 0$. This proves (28).

As in [12, we get that

$$
d\left(x_{\epsilon}, \partial \Omega\right)=(1+o(1))\left|x_{1, \epsilon}\right|
$$

when $\epsilon \rightarrow 0$. We write that

$$
\pi\left(x_{\epsilon}\right)=\pi\left(x_{1, \epsilon}+\varphi_{0}\left(y_{\epsilon}, z_{\epsilon}\right), y_{\epsilon}, z_{\epsilon}\right)=\left(x_{1, \epsilon}+\varphi_{0}\left(y_{\epsilon}, z_{\epsilon}\right), y_{\epsilon}, 0\right) .
$$

With (201) and (28), we then get that

$$
\varphi_{0}\left(y_{\epsilon}, z_{\epsilon}\right)=O\left(k_{\epsilon}\right) \text { and } y_{\epsilon}=O\left(k_{\epsilon}\right)
$$

when $\epsilon \rightarrow 0$. Noting that $\varphi_{0}\left(y_{\epsilon}, z_{\epsilon}\right)=\varphi_{0}\left(0, z_{\epsilon}\right)+O\left(\left|y_{\epsilon}\right|\right)$ when $\epsilon \rightarrow 0$, we get that $\varphi_{0}\left(0, z_{\epsilon}\right)=O\left(k_{\epsilon}\right)$. These last equalities, (28), (29) and (30) prove (27).

We let

$$
\lambda_{\epsilon}:=-\frac{x_{1, \epsilon}}{k_{\epsilon}}>0, \theta_{\epsilon}:=\frac{y_{\epsilon}}{k_{\epsilon}} \in \mathcal{P} \text { and } \rho_{\epsilon}:=-\frac{\varphi_{0}\left(0, z_{\epsilon}\right)}{k_{\epsilon}} .
$$

It follows from (27) and (29) that there exist $\lambda_{0} \geq 0, \rho_{0} \in \mathbb{R}$ and $\theta_{0} \in \mathcal{P}$ such that

$$
\lambda_{0}:=\lim _{\epsilon \rightarrow 0} \lambda_{\epsilon}, \theta_{0}:=\lim _{\epsilon \rightarrow 0} \theta_{\epsilon} \text { and } \rho_{0}:=\lim _{\epsilon \rightarrow 0} \rho_{\epsilon} .
$$

We claim that $\rho_{\epsilon} \geq 0$ for all $\epsilon>0$. Indeed, since $\mathcal{P}^{\perp} \cap \Omega=\emptyset$, there exists $\delta>0$ such that for all $z \in \operatorname{span}\left\{\vec{e}_{k+1}, \ldots, \vec{e}_{n}\right\} \cap B_{\delta}(0)$,

$$
\varphi_{0}(0, z) \leq 0 \text {. }
$$

The definition (31) of $\rho_{\epsilon}$ yields that $\rho_{\epsilon} \geq 0$ for all $\epsilon>0$. Note that it follows from (33) that there exists $C>0$ such that

$$
d(x, \partial \Omega) \leq C|\pi(x)|
$$

for all $x \in \Omega$.

Step 3.4. From now on, we let $\bar{z}_{\epsilon}=\left(0,0, z_{\epsilon}\right)$ for all $\epsilon>0$ where $z_{\epsilon}$ is defined in (26), and for any $x \in \frac{U-\bar{z}_{\epsilon}}{k_{\epsilon}} \cap\left\{x_{1} \leq 0\right\}$ we set

$$
v_{\epsilon}(x):=\frac{u_{\epsilon} \circ \varphi\left(\bar{z}_{\epsilon}+k_{\epsilon} x\right)}{u_{\epsilon}\left(x_{\epsilon}\right)},
$$

where $\varphi$ is defined in (13). It follows from (31) that

$$
v_{\epsilon}\left(-\lambda_{\epsilon}, \theta_{\epsilon}, 0\right)=1 \text {. }
$$

As is easily checked, for any $\eta \in C_{c}^{\infty}\left(\mathbb{R}^{n}\right)$ we have that $\eta v_{\epsilon} \in H_{1,0}^{2}\left(\mathbb{R}_{-}^{n}\right)$ for all $\epsilon>0$.

Step 3.4.1. There exists $v \in H_{1,0}^{2}\left(\mathbb{R}_{-}^{n}\right)$ such that for any $\eta \in C_{c}^{\infty}\left(\mathbb{R}^{n}\right)$,

$$
\eta v_{\epsilon} \rightarrow \eta v
$$

weakly in $H_{1,0}^{2}\left(\mathbb{R}_{-}^{n}\right)$ when $\epsilon \rightarrow 0$. The proof is rather similar to what was done in 12. to which we refer for details. 
Step 3.4.2. We claim that $\lim _{\epsilon \rightarrow 0} v_{\epsilon}=v$ in $C_{l o c}^{1}\left(\overline{\mathbb{R}_{-}^{n}}\right)$, where $v \not \equiv 0$.

Indeed, let $R>0$, and for any $i, j=1, \ldots, n$, we let

$$
\left(\tilde{g}_{\epsilon}\right)_{i j}=\left(\partial_{i} \varphi\left(\bar{z}_{\epsilon}+k_{\epsilon} x\right), \partial_{j} \varphi\left(\bar{z}_{\epsilon}+k_{\epsilon} x\right)\right)
$$

where $(\cdot, \cdot)$ denotes the Euclidean scalar product on $\mathbb{R}^{n}$. We consider $\tilde{g}_{\epsilon}$ as a metric on $\mathbb{R}^{n}$. We let

$$
\Delta_{\tilde{g}_{\epsilon}}=-\tilde{g}_{\epsilon}^{i j}\left(\partial_{i j}-\Gamma_{i j}^{k}\left(\tilde{g}_{\epsilon}\right) \partial_{k}\right),
$$

where $\tilde{g}_{\epsilon}^{i j}:=\left(\tilde{g}_{\epsilon}^{-1}\right)_{i j}$ are the coordinates of the inverse of the tensor $\tilde{g}_{\epsilon}$ and the $\Gamma_{i j}^{k}\left(\tilde{g}_{\epsilon}\right)$ 's are the Christoffel symbols of the metric $\tilde{g}_{\epsilon}$. With a change of variable and the definition (35), equation (15) rewrites as

$$
\Delta_{\tilde{g}_{\epsilon}} v_{\epsilon}+k_{\epsilon}^{2} a_{\epsilon} \circ \varphi\left(\bar{z}_{\epsilon}+k_{\epsilon} x\right) v_{\epsilon}=\frac{v_{\epsilon}^{2^{\star}-1-p_{\epsilon}}}{\left|\frac{\pi\left(\varphi\left(\bar{z}_{\epsilon}+k_{\epsilon} x\right)\right)}{k_{\epsilon}}\right|^{s}} \text { in } \mathcal{D}^{\prime}\left(\left\{x_{1}<0\right\}\right)
$$

for all $\epsilon>0$. It follows from the definition (13) of $\varphi$ and (33) that there exists $C_{R}>0$ such that $\left|\pi\left(\varphi\left(\bar{z}_{\epsilon}+k_{\epsilon} x\right)\right)\right| \geq C_{R} k_{\epsilon}|\pi(x)|$ for all $x \in \mathbb{R}_{-}^{n} \cap B_{R}(0)$. With (18) and (35), we get that $0 \leq v_{\epsilon} \leq 1$. With the method used in the Appendix, we get that $\left(v_{\epsilon}\right)_{\epsilon>0}$ converges in $C_{l o c}^{1}\left(\mathbb{R}_{-}^{n}\right)$. Since $v_{\epsilon} \rightarrow v$ weakly in $H_{1,0}^{2}\left(\mathbb{R}_{-}^{n}\right)$ when $k \rightarrow+\infty$, we get that $\lim _{\epsilon \rightarrow 0} v_{\epsilon}=v$ in $C_{l o c}^{1}\left(\overline{\mathbb{R}_{-}^{n}}\right)$. With (36) and (32), we get that $v\left(-\lambda_{0}, \theta_{0}, 0\right)=1$, and in particular, $v \neq \equiv$ and $\lambda_{0}>0$.

Step 3.4.3. We claim that $\Delta v=\frac{v^{2^{\star}-1}}{|\pi(x)|^{s}}$ in $\mathcal{D}^{\prime}\left(\mathbb{R}_{-}^{n}\right)$ and that

$$
\int_{\mathbb{R}_{-}^{n}}|\nabla v|^{2} d x=\mu_{s, \mathcal{P}}(\Omega)^{\frac{2^{\star}}{2^{\star}-2}}=\mu_{s, \mathcal{P}}\left(\mathbb{R}_{-}^{n}\right)^{\frac{2^{\star}}{2^{\star}-2}} .
$$

Indeed, by passing to the weak limit $\epsilon \rightarrow 0$ in (37), we get that

$$
\Delta v=\frac{v^{2^{\star}-1}}{\left|\pi(x)-\left(\rho_{0}, 0,0\right)\right|^{s}} \text { in } \mathcal{D}^{\prime}\left(\mathbb{R}_{-}^{n}\right) .
$$

Testing this equality with $v \in H_{1,0}^{2}\left(\mathbb{R}_{-}^{n}\right)$ and using the optimal Hardy-Sobolev inequality (6), we get that

$$
\begin{aligned}
\left(\int_{\mathbb{R}_{-}^{n}}|\nabla v|^{2} d x\right)^{\frac{2^{\star}-2}{2^{\star}}} & =\frac{\int_{\mathbb{R}_{-}^{n}}|\nabla v|^{2} d x}{\left(\int_{\mathbb{R}_{-}^{n}} \frac{v^{2^{\star}}}{\left|\pi(x)-\left(\rho_{0}, 0,0\right)\right|^{s}} d x\right)^{\frac{2}{2^{\star}}}} \\
& \geq \frac{\int_{\mathbb{R}_{-}^{n}}|\nabla v|^{2} d x}{\left(\int_{\mathbb{R}_{-}^{n}} \frac{v^{2^{\star}}}{|\pi(x)|^{s}} d x\right)^{\frac{2}{2^{\star}}} \geq \mu_{s, \mathcal{P}}\left(\mathbb{R}_{-}^{n}\right) .} .
\end{aligned}
$$

Here, we have used the fact that $\left|\pi(x)-\left(\rho_{0}, 0,0\right)\right| \geq|\pi(x)|$ since $\rho_{0} \geq 0$ and $x_{1}<0$ for all $x \in \mathbb{R}_{-}^{n}$. We then obtain that

$$
\int_{\mathbb{R}_{-}^{n}}|\nabla v|^{2} d x \geq \mu_{s, \mathcal{P}}\left(\mathbb{R}_{-}^{n}\right)^{\frac{2^{\star}}{2^{\star}-2}}
$$

Moreover, see for instance [12], we have that $\int_{\mathbb{R}_{-}^{n}}|\nabla v|^{2} d x \leq \mu_{s, \mathcal{P}}\left(\mathbb{R}_{-}^{n}\right)^{\frac{2^{\star}}{2^{\star}-2}}$. We then get that

$$
\int_{\mathbb{R}_{-}^{n}}|\nabla v|^{2} d x=\mu_{s, \mathcal{P}}(\Omega)^{\frac{2^{\star}}{2^{\star}-2}}=\mu_{s, \mathcal{P}}\left(\mathbb{R}_{-}^{n}\right)^{\frac{2^{\star}}{2^{\star}-2}}
$$


and that

$$
\lim _{\epsilon \rightarrow 0} \rho_{\epsilon}=\rho_{0}=0 \text { and } \lim _{\epsilon \rightarrow 0} \mu_{\epsilon}^{p_{\epsilon}}=1 .
$$

For this last assertion, we refer to [12].

Proposition 3.3 now follows from Steps 31 to 3.4 .

We shall also need the following two claims for the next section

Step 3.5. Under the hypothesis of Proposition 3.3, we have that

$$
\lim _{R \rightarrow+\infty} \lim _{\epsilon \rightarrow 0} \int_{\Omega \backslash B_{R k_{\epsilon}}\left(\varphi\left(\bar{z}_{\epsilon}\right)\right)} \frac{u_{\epsilon}^{2^{\star}-p_{\epsilon}}}{|\pi(x)|^{s}} d x=0 .
$$

We omit the proof, which is quite similar to [12.

Step 3.6. We also claim that

$$
\lim _{\epsilon \rightarrow 0} u_{\epsilon}=0 \text { in } C_{l o c}^{1}\left(\bar{\Omega} \backslash\left\{x_{0}\right\}\right) .
$$

Indeed, for $\delta>0$, it follows from (41) that

$$
\lim _{\epsilon \rightarrow 0} \int_{\Omega \backslash B_{\delta}\left(x_{0}\right)} \frac{u_{\epsilon}^{2^{\star}-1-p_{\epsilon}}(x)}{|\pi(x)|^{s}} d x=0 .
$$

Using the techniques in the Appendix of [12, 13], we get that

$$
\lim _{\epsilon \rightarrow 0}\left\|u_{\epsilon}\right\|_{L^{p}\left(\Omega \backslash B_{\delta}\left(x_{0}\right)\right)}=0
$$

for all $p \geq 1$, and by the method developed in this paper's Appendix, we get (42).

\section{Blow-up analysis, Part II}

This section is devoted to the proof of the following strong pointwise estimate.

Proposition 4.1. Let $\Omega$ be a smooth bounded domain of $\mathbb{R}^{n}, n \geq 3$, and let $\mathcal{P}$ be a linear subspace of $\mathbb{R}^{n}$ such that $2 \leq \operatorname{dim}_{\mathbb{R}} \mathcal{P} \leq n-1$. Let $s \in(0,2)$ and assume that (10) holds. For $\left(p_{\epsilon}\right)_{\epsilon>0}$ in $\left[0,2^{\star}-2\right)$ such that $\lim _{\epsilon \rightarrow 0} p_{\epsilon}=0$ and $\left(a_{\epsilon}\right)_{\epsilon>0}$ as in (14), we consider $\left(u_{\epsilon}\right)_{\epsilon>0} \in H_{1,0}^{2}(\Omega) \cap C^{2}\left(\bar{\Omega} \backslash \mathcal{P}^{\perp}\right)$ such that (15), (16) and (17) hold. We let $x_{0}, \varphi,\left(\mu_{\epsilon}\right)_{\epsilon>0}$ and $\left(\bar{z}_{\epsilon}\right)_{\epsilon>0}$ be as in Proposition 3.3. Then, there exists $C>0$ such that

$$
u_{\epsilon}(x) \leq C d(x, \partial \Omega)+C \frac{\mu_{\epsilon}^{\frac{n}{2}} d(x, \partial \Omega)}{\left(\mu_{\epsilon}^{2}+\left|x-\varphi\left(\bar{z}_{\epsilon}\right)\right|^{2}\right)^{\frac{n}{2}}}
$$

and

$$
\left|\nabla u_{\epsilon}(x)\right| \leq C+C \frac{\mu_{\epsilon}^{\frac{n}{2}}}{\left(\mu_{\epsilon}^{2}+\left|x-\varphi\left(\bar{z}_{\epsilon}\right)\right|^{2}\right)^{\frac{n}{2}}}
$$

for all $\epsilon>0$ and all $x \in \Omega$.

Proof. We take inspiration from [8]. We proceed in five steps.

Step 4.1. We claim that there exists $C>0$ such that

$$
|\pi(x)|^{\frac{n-2}{2}} u_{\epsilon}(x)^{1-\frac{p_{\epsilon}}{2^{\star}-2}} \leq C
$$

for all $\epsilon>0$ and all $x \in \Omega$. 
Indeed, if not, we let $y_{\epsilon} \in \Omega$ such that

$$
\left|\pi\left(y_{\epsilon}\right)\right|^{\frac{n-2}{2}} u_{\epsilon}\left(y_{\epsilon}\right)^{1-\frac{p_{\epsilon}}{2^{\star}-2}}=\sup _{x \in \Omega}|\pi(x)|^{\frac{n-2}{2}} u_{\epsilon}(x)^{1-\frac{p_{\epsilon}}{2^{\star}-2}} \rightarrow+\infty
$$

as $\epsilon \rightarrow 0$. We then let

$$
\nu_{\epsilon}:=u_{\epsilon}\left(y_{\epsilon}\right)^{-\frac{2}{n-2}} \text { and } \ell_{\epsilon}:=\nu_{\epsilon}^{1-\frac{p_{\epsilon}}{2^{\star}-2}}
$$

for all $\epsilon>0$. It follows from (46) and (47) that

$$
\lim _{\epsilon \rightarrow 0} \nu_{\epsilon}=0 \text { and } \lim _{\epsilon \rightarrow 0} \frac{\left|\pi\left(y_{\epsilon}\right)\right|}{\ell_{\epsilon}}=+\infty,
$$

and from (18) and (40) that

$$
\lim _{\epsilon \rightarrow 0} \nu_{\epsilon}^{p_{\epsilon}}=1
$$

We also let

$$
\gamma_{\epsilon}:=\left|\pi\left(y_{\epsilon}\right)\right|^{\frac{s}{2}}\left|u_{\epsilon}\left(y_{\epsilon}\right)\right|^{\frac{2-2^{\star}+p_{\epsilon}}{2}},
$$

for all $\epsilon>0$. It follows from (48) that

$$
\lim _{\epsilon \rightarrow 0} \frac{\gamma_{\epsilon}}{\left|\pi\left(y_{\epsilon}\right)\right|}=0
$$

Case 4.1.1. We assume first that, up to a subsequence, there exists $\rho>0$ such that

$$
\frac{d\left(y_{\epsilon}, \partial \Omega\right)}{\gamma_{\epsilon}} \geq 3 \rho
$$

for all $\epsilon>0$. For any $x \in B_{2 \rho}(0)$ and any $\epsilon>0$, we let

$$
w_{\epsilon}(x):=\nu_{\epsilon}^{\frac{n-2}{2}} u_{\epsilon}\left(y_{\epsilon}+\gamma_{\epsilon} x\right) \text {. }
$$

Note that $w_{\epsilon}$ is well defined thanks to (52). With (46) and (50), we get that

$$
\left|\frac{\pi\left(y_{\epsilon}\right)}{\left|\pi\left(y_{\epsilon}\right)\right|}+\frac{\gamma_{\epsilon}}{\left|\pi\left(y_{\epsilon}\right)\right|} \pi(x)\right|^{\frac{n-2}{2}} w_{\epsilon}(x)^{1-\frac{p_{\epsilon}}{2^{\star}-2}} \leq 1 .
$$

In particular, with (51), there exists $C_{0}>0$ such that

$$
0 \leq w_{\epsilon}(x) \leq C_{0}
$$

for all $x \in B_{2 \rho}(0)$ and all $\epsilon>0$. With (15), we get that

$$
\Delta w_{\epsilon}+\gamma_{\epsilon}^{2} a_{\epsilon}\left(y_{\epsilon}+\gamma_{\epsilon} x\right) w_{\epsilon}=\frac{w_{\epsilon}^{2^{\star}-1-p_{\epsilon}}}{\left|\frac{\pi\left(y_{\epsilon}\right)}{\left|\pi\left(y_{\epsilon}\right)\right|}+\frac{\gamma_{\epsilon}}{\left|\pi\left(y_{\epsilon}\right)\right|} \pi(x)\right|^{s}}
$$

for all $x \in B_{2 \rho}(0)$ and all $\epsilon>0$. Since (48) and (54) hold, it follows from standard elliptic theory that there exists $w \in C^{1}\left(B_{2 \rho}(0)\right)$ such that $w(0)=1$ and

$$
\lim _{\epsilon \rightarrow 0} w_{\epsilon}=w
$$

in $C_{l o c}^{1}\left(B_{2 \rho}(0)\right)$. Mimicking what was done in Step 3,1 , we get a contradiction.

Case 4.1.2. We assume that

$$
\lim _{\epsilon \rightarrow 0} \frac{d\left(y_{\epsilon}, \partial \Omega\right)}{\gamma_{\epsilon}}=0 .
$$

As in Step 31, we get a contradiction. We refer to [12] for proof in a similar context.

In both cases, we have contradicted (46). This proves (45). 
Step 4.2. This step is a slight improvement of (45). We claim that

$$
\lim _{R \rightarrow+\infty} \lim _{\epsilon \rightarrow 0} \sup _{x \in \Omega \backslash B_{R k_{\epsilon}}\left(\varphi\left(\bar{z}_{\epsilon}\right)\right)}|\pi(x)|^{\frac{n-2}{2}} u_{\epsilon}(x)^{1-\frac{p_{\epsilon}}{2^{\star}-2}}=0 .
$$

The proof is similar to Step 4,1 and uses the techniques developed in [12. We refer to Step 41 and 12 for the details.

Step 4.3. We claim that for any $\nu \in(0,1)$ and any $R>0$, there exists $C(\nu, R)>0$ such that

$$
u_{\epsilon}(x) \leq C(\nu, R) \cdot\left(\frac{\mu_{\epsilon}^{\frac{n}{2}-\nu(n-1)} d(x, \partial \Omega)^{1-\nu}}{\left(\mu_{\epsilon}^{2}+\left|x-\varphi\left(\bar{z}_{\epsilon}\right)\right|^{2}\right)^{\frac{n(1-\nu)}{2}}}+d(x, \partial \Omega)^{1-\nu}\right)
$$

for all $x \in \Omega$ and all $\epsilon>0$.

Indeed, let $G$ be the Green's function for $\Delta$ in $\Omega$ with Dirichlet boundary condition, and set $H_{\epsilon}(x)=-\partial_{\vec{n}} G\left(x, \varphi\left(\bar{z}_{\epsilon}\right)\right)$ for all $x \in \bar{\Omega} \backslash\left\{\varphi\left(\bar{z}_{\epsilon}\right)\right\}$, where here $\vec{n}$ denotes the outward normal vector at $\partial \Omega$. It follows from Theorem 9.2 of [13] that $H_{\epsilon} \in C^{2}\left(\bar{\Omega} \backslash\left\{\varphi\left(\bar{z}_{\epsilon}\right)\right\}\right)$, that

$$
\Delta H_{\epsilon}=0
$$

in $\Omega$ and that there exist $\delta_{1}, C_{1}>0$ such that

$$
\frac{d(x, \partial \Omega)}{C_{1}\left|x-\varphi\left(\bar{z}_{\epsilon}\right)\right|^{n}} \leq H_{\epsilon}(x) \leq \frac{C_{1} d(x, \partial \Omega)}{\left|x-\varphi\left(\bar{z}_{\epsilon}\right)\right|^{n}}
$$

and, using (34), that

$$
\frac{\left|\nabla H_{\epsilon}(x)\right|}{H_{\epsilon}(x)} \geq \frac{1}{C_{1}^{\prime} d(x, \partial \Omega)} \geq \frac{1}{C_{1}|\pi(x)|}
$$

for all $x \in \Omega \cap B_{2 \delta_{1}}(0)$. Let $\lambda_{1}>0$ be the first eigenvalue of $\Delta$ on $\Omega$, and let $\psi \in C^{2}(\bar{\Omega})$ be "the first eigenfunction" in such a way that

$$
\begin{cases}\Delta \psi=\lambda_{1} \psi & \text { in } \Omega \\ \psi>0 & \text { in } \Omega \\ \psi=0 & \text { on } \partial \Omega\end{cases}
$$

It follows from standard elliptic theory, Hopf's maximum principle and again (34) that there exists $C_{2}, \delta_{2}>0$ such that

$$
\frac{1}{C_{2}} d(x, \partial \Omega) \leq \psi(x) \leq C_{2} d(x, \partial \Omega) \text { and } \frac{|\nabla \psi(x)|}{\psi(x)} \geq \frac{1}{C_{2}^{\prime} d(x, \partial \Omega)} \geq \frac{1}{C_{2}|\pi(x)|}
$$

for all $x \in \Omega \cap B_{2 \delta_{2}}\left(\varphi\left(\bar{z}_{\epsilon}\right)\right)$. We now consider the operator

$$
L_{\epsilon}=\Delta+\left(a_{\epsilon}-\frac{u_{\epsilon}^{2^{\star}-2-p_{\epsilon}}}{|\pi(x)|^{s}}\right) .
$$

Step 4.3.1. We claim that there exist $\delta_{0}>0$ and $R_{0}>0$ such that for any $\nu \in(0,1)$ and any $R>R_{0}, \delta \in\left(0, \delta_{0}\right)$, we have that

$$
L_{\epsilon} H_{\epsilon}^{1-\nu}>0 \text {, and } L_{\epsilon} \psi^{1-\nu}>0
$$

for all $x \in \Omega \cap B_{\delta}\left(\varphi\left(\bar{z}_{\epsilon}\right)\right) \backslash \bar{B}_{R k_{\epsilon}}\left(\varphi\left(\bar{z}_{\epsilon}\right)\right)$ and for all $\epsilon>0$ sufficiently small.

Indeed, with (59), we get that

$$
\frac{L_{\epsilon} H_{\epsilon}^{1-\nu}}{H_{\epsilon}^{1-\nu}}(x)=a_{\epsilon}(x)+\nu(1-\nu) \frac{\left|\nabla H_{\epsilon}\right|^{2}}{H_{\epsilon}^{2}}(x)-\frac{u_{\epsilon}(x)^{2^{\star}-2-p_{\epsilon}}}{|\pi(x)|^{s}}
$$


for all $x \in \Omega$ and all $\epsilon>0$. We let $\alpha>0$. It follows from (57) that there exists $R_{0}>0$ such that for any $R>R_{0}$, we have that

$$
|\pi(x)|^{2-s}\left|u_{\epsilon}(x)\right|^{2^{\star}-2-p_{\epsilon}}<\alpha
$$

for all $x \in\left(B_{\delta}\left(\varphi\left(\bar{z}_{\epsilon}\right)\right) \backslash \bar{B}_{R k_{\epsilon}}\left(\varphi\left(\bar{z}_{\epsilon}\right)\right)\right) \cap \Omega$ and all $\epsilon>0$ small enough. With (14), (64) and (61), we get that for $\alpha>0$ and $\delta>0$ small enough, we have that

$$
\frac{L_{\epsilon} H_{\epsilon}^{1-\nu}}{H_{\epsilon}^{1-\nu}}(x)>\frac{\nu(1-\nu)-\alpha C_{1}^{2}-C_{1}^{2}|\pi(x)|^{2}\left|a_{\epsilon}(x)\right|}{C_{1}^{2}|\pi(x)|^{2}}>0
$$

for all $x \in\left(B_{\delta}\left(\varphi\left(\bar{z}_{\epsilon}\right)\right) \backslash \bar{B}_{R k_{\epsilon}}\left(\varphi\left(\bar{z}_{\epsilon}\right)\right)\right) \cap \Omega$ and all $\epsilon>0$ small enough. The proof of the second inequality of (63) follows in the same way.

Step 4.3.2. It follows from (19) in Proposition 3.3 that there exists $C_{1}(R)>0$ such that

$$
u_{\epsilon}(x) \leq C_{1}(R) \mu_{\epsilon}^{-\frac{n}{2}} d(x, \partial \Omega)
$$

for all $x \in \Omega \cap \partial B_{R k_{\epsilon}}\left(\varphi\left(\bar{z}_{\epsilon}\right)\right)$ and all $\epsilon>0$. In particular, there exists $C(R)>0$ such that

$$
u_{\epsilon}(x) \leq C(R) \mu_{\epsilon}^{\frac{n}{2}-\nu(n-1)} H_{\epsilon}^{1-\nu}(x)
$$

for all $x \in \Omega \cap \partial B_{R k_{\epsilon}}\left(\varphi\left(\bar{z}_{\epsilon}\right)\right)$ and all $\epsilon>0$.

It follows from (42) that there exists $C_{1}(\delta)>0$ such that

$$
u_{\epsilon}(x) \leq C_{1}(\delta) d(x, \partial \Omega)
$$

for all $x \in \Omega \cap \partial B_{\delta}\left(\varphi\left(\bar{z}_{\epsilon}\right)\right)$ and all $\epsilon>0$. In particular, there exists $C(\delta)>0$ such that $u_{\epsilon}(x) \leq C(\delta) \psi(x)^{1-\nu}$ for all $x \in \Omega \cap \partial B_{\delta}\left(\varphi\left(\bar{z}_{\epsilon}\right)\right)$ and all $\epsilon>0$. We let

$$
D_{\epsilon, R, \delta}:=\left(B_{\delta}\left(\varphi\left(\bar{z}_{\epsilon}\right)\right) \backslash \bar{B}_{R k_{\epsilon}}\left(\varphi\left(\bar{z}_{\epsilon}\right)\right)\right) \cap \Omega .
$$

It follows from (65) and (66) that

$$
u_{\epsilon}(x) \leq C(R) \mu_{\epsilon}^{\frac{n}{2}-\nu(n-1)} H_{\epsilon}^{1-\nu}(x)+C(\delta) \psi(x)^{1-\nu}
$$

for all $\epsilon>0$ and all $x \in \partial D_{\epsilon, R, \delta}$.

Step 4.3.3. We claim that $L_{\epsilon}$ is coercive and therefore verifies the comparison principle on $D_{\epsilon, R, \delta}$.

Indeed, with (41), we get that for any $\alpha>0$, there exists $\tilde{R}_{0}>0$ such that for any $R>\tilde{R}_{0}$, we have that

$$
\int_{\Omega \backslash B_{R k_{\epsilon}}\left(\varphi\left(\bar{z}_{\epsilon}\right)\right)} \frac{u_{\epsilon}^{2^{\star}-p_{\epsilon}}(x)}{|\pi(x)|^{s}} d x \leq \alpha .
$$

Since $\Delta+a_{\epsilon}$ is uniformly coercive, we get that $L_{\epsilon}$ is coercive on $\Omega \backslash B_{R k_{\epsilon}}\left(\varphi\left(\bar{z}_{\epsilon}\right)\right)$ for $R$ large enough. We refer to Lemma 3.4 of 21 for details on this assertion.

Step 4.3.4. Since

$$
L_{\epsilon}\left(C(R) \mu_{\epsilon}^{\frac{n}{2}-\nu(n-1)} H_{\epsilon}^{1-\nu}(x)+C(\delta) \psi(x)^{1-\nu}\right)>0=L_{\epsilon} u_{\epsilon}
$$

in $D_{\epsilon, R, \delta}$ and (67) holds, we get from Step 4.3 .3 that

$$
u_{\epsilon}(x) \leq C(R) \mu_{\epsilon}^{\frac{n}{2}-\nu(n-1)} H_{\epsilon}^{1-\nu}(x)+C(\delta) \psi(x)^{1-\nu}
$$

for all $x \in D_{\epsilon, R, \delta}$. With (60) and (62), we then get that (58) holds on $D_{\epsilon, R, \delta}=$ $\left(B_{\delta}\left(\varphi\left(\bar{z}_{\epsilon}\right)\right) \backslash \bar{B}_{R k_{\epsilon}}\left(\varphi\left(\bar{z}_{\epsilon}\right)\right)\right) \cap \Omega$ for $R$ large and $\delta$ small. It follows from this last assertion, (19) in Proposition 3.3 and (42) that (58) holds on $\Omega$. 
Step 4.4. We claim that there exists $C>0$ such that

$$
u_{\epsilon}(x) \leq C d(x, \partial \Omega)+C \frac{\mu_{\epsilon}^{\frac{n}{2}} d(x, \partial \Omega)}{\left(\mu_{\epsilon}^{2}+\left|x-\varphi\left(\bar{z}_{\epsilon}\right)\right|^{2}\right)^{\frac{n}{2}}}
$$

for all $x \in \Omega$ and all $\epsilon>0$.

Indeed, it follows from (19) in Proposition 3.3 and (42) that for any $\delta, R>0$, inequality (68) holds for all $x \in\left(\Omega \backslash B_{\delta}\left(\varphi\left(\bar{z}_{\epsilon}\right)\right)\right) \cup\left(\Omega \cap B_{R \mu_{\epsilon}}\left(\varphi\left(\bar{z}_{\epsilon}\right)\right)\right)$ for all $\epsilon>0$. What is left is to prove (68) for any sequence $\left(y_{\epsilon}\right)_{\epsilon>0} \in \Omega$ such that

$$
\lim _{\epsilon \rightarrow 0} y_{\epsilon}=x_{0} \text { and } \lim _{\epsilon \rightarrow 0} \frac{\left|y_{\epsilon}-\varphi\left(\bar{z}_{\epsilon}\right)\right|}{k_{\epsilon}}=+\infty \text {. }
$$

We show that (68) holds for $x=y_{\epsilon}$. With Green's representation formula, we get that

$$
u_{\epsilon}\left(y_{\epsilon}\right)=\int_{\Omega} G_{\epsilon}\left(y_{\epsilon}, y\right) \frac{u_{\epsilon}(y)^{2^{\star}-1-p_{\epsilon}}}{|\pi(y)|^{s}} d y,
$$

where $G_{\epsilon}$ is the Green's function for the uniformly coercive operator $\Delta+a_{\epsilon}$. For $\nu \in(0,1)$, we use (58) and (34) to get that

$$
\begin{aligned}
u_{\epsilon}\left(y_{\epsilon}\right) \leq & C \int_{\Omega} G_{\epsilon}\left(y_{\epsilon}, y\right) \frac{d(y, \partial \Omega)^{(1-\nu)\left(2^{\star}-1-p_{\epsilon}\right)}}{|\pi(y)|^{s}} d y \\
& +C \int_{\Omega} \frac{G_{\epsilon}\left(y_{\epsilon}, y\right)}{|\pi(y)|^{s}}\left(\frac{\mu_{\epsilon}^{\frac{n}{2}-(n-1) \nu} d(y, \partial \Omega)^{1-\nu}}{\left(\mu_{\epsilon}^{2}+\left|y-\varphi\left(\bar{z}_{\epsilon}\right)\right|^{2}\right)^{\frac{n(1-\nu)}{2}}}\right)^{2^{\star}-1-p_{\epsilon}} d y \\
\leq & I_{\epsilon, 1}+I_{\epsilon, 2}+I_{\epsilon, 3},
\end{aligned}
$$

where

$$
\begin{gathered}
I_{\epsilon, 1}:=\int_{\Omega} G_{\epsilon}\left(y_{\epsilon}, y\right)|\pi(y)|^{(1-\nu)\left(2^{\star}-1-p_{\epsilon}\right)-s} d y \\
I_{\epsilon, 2}:=\int_{D_{\epsilon, 2}} \frac{G_{\epsilon}\left(y_{\epsilon}, y\right)}{|\pi(y)|^{s}}\left(\frac{\mu_{\epsilon}^{\frac{n}{2}-(n-1) \nu} d(y, \partial \Omega)^{1-\nu}}{\left(\mu_{\epsilon}^{2}+\left|y-\varphi\left(\bar{z}_{\epsilon}\right)\right|^{2}\right)^{\frac{n(1-\nu)}{2}}}\right)^{2^{\star}-1-p_{\epsilon}} d y
\end{gathered}
$$

and

$$
I_{\epsilon, 3}:=\int_{D_{\epsilon, 3}} \frac{G_{\epsilon}\left(y_{\epsilon}, y\right)}{|\pi(y)|^{s}}\left(\frac{\mu_{\epsilon}^{\frac{n}{2}-(n-1) \nu} d(y, \partial \Omega)^{1-\nu}}{\left(\mu_{\epsilon}^{2}+\left|y-\varphi\left(\bar{z}_{\epsilon}\right)\right|^{2}\right)^{\frac{n(1-\nu)}{2}}}\right)^{2^{\star}-1-p_{\epsilon}} d y
$$

for all $\epsilon>0$, where

$$
D_{\epsilon, 2}:=\left\{\left|y_{\epsilon}-y\right|>\frac{1}{2}\left|y_{\epsilon}-\varphi\left(\bar{z}_{\epsilon}\right)\right|\right\} \text { and } D_{\epsilon, 3}:=\left\{\left|y_{\epsilon}-y\right|<\frac{1}{2}\left|y_{\epsilon}-\varphi\left(\bar{z}_{\epsilon}\right)\right|\right\} .
$$

We first deal with $I_{\epsilon, 1}$. The Green's function verifies

$$
G_{\epsilon}\left(y_{\epsilon}, y\right) \leq C \frac{d\left(y_{\epsilon}, \partial \Omega\right)}{\left|y_{\epsilon}-y\right|^{n-1}}
$$

for all $y \in \Omega \backslash\left\{y_{\epsilon}\right\}$ and all $\epsilon>0$. We refer to [13] for the proof of this assertion. Since $s \in(0,2)$ and $\varphi\left(\bar{z}_{\epsilon}\right) \in \partial \Omega$, we then get that

$$
I_{\epsilon, 1} \leq C d\left(y_{\epsilon}, \partial \Omega\right) \int_{\Omega} \frac{|\pi(y)|^{(1-\nu)\left(2^{\star}-1-p_{\epsilon}\right)-s}}{\left|y_{\epsilon}-y\right|^{n-1}} \leq C d\left(y_{\epsilon}, \partial \Omega\right)
$$

for all $\epsilon>0$. 
For $I_{\epsilon, 2}$, we note that the Green's function verifies

$$
G_{\epsilon}\left(y_{\epsilon}, y\right) \leq C \frac{d\left(y_{\epsilon}, \partial \Omega\right) d(y, \partial \Omega)}{\left|y_{\epsilon}-y\right|^{n}}
$$

for all $y \in \Omega \backslash\left\{y_{\epsilon}\right\}$ and all $\epsilon>0$. We again refer to [13 for the proof of this assertion. We then get with (34) and a change of variables that

$$
\begin{aligned}
& I_{\epsilon, 2} \\
& \leq C \int_{D_{\epsilon, 2}} \frac{d\left(y_{\epsilon}, \partial \Omega\right)}{\left|y_{\epsilon}-y\right|^{n}} \frac{\mu_{\epsilon}^{\left(\frac{n}{2}-(n-1) \nu\right)\left(2^{\star}-1-p_{\epsilon}\right)} d(y, \partial \Omega)^{(1-\nu)\left(2^{\star}-1-p_{\epsilon}\right)+1-s}}{\left(\mu_{\epsilon}^{2}+\left|x-\varphi\left(\bar{z}_{\epsilon}\right)\right|^{2}\right)^{\frac{n(1-\nu)}{2}\left(2^{\star}-1-p_{\epsilon}\right)}} d y \\
& \leq C \frac{d\left(y_{\epsilon}, \partial \Omega\right) \mu_{\epsilon}^{\left(\frac{n}{2}-(n-1) \nu\right)\left(2^{\star}-1-p_{\epsilon}\right)}}{\left|y_{\epsilon}-\varphi\left(\bar{z}_{\epsilon}\right)\right|^{n}} \int_{\Omega} \frac{\left|y-\varphi\left(\bar{z}_{\epsilon}\right)\right|^{(1-\nu)\left(2^{\star}-1-p_{\epsilon}\right)+1-s}}{\left(\mu_{\epsilon}^{2}+\left|x-\varphi\left(\bar{z}_{\epsilon}\right)\right|^{2}\right)^{\frac{n(1-\nu)}{2}\left(2^{\star}-1-p_{\epsilon}\right)}} d y \\
& \leq C \frac{d\left(y_{\epsilon}, \partial \Omega\right) \mu_{\epsilon}^{\frac{n}{2}}}{\left|y_{\epsilon}-\varphi\left(\bar{z}_{\epsilon}\right)\right|^{n}} \int_{\mathbb{R}^{n}} \frac{|z|^{(1-\nu)\left(2^{\star}-1-p_{\epsilon}\right)+1-s}}{\left(1+|z|^{2}\right)^{\frac{n(1-\nu)}{2}\left(2^{\star}-1-p_{\epsilon}\right)}} d y \leq C \frac{d\left(y_{\epsilon}, \partial \Omega\right) \mu_{\epsilon}^{\frac{n}{2}}}{\left|y_{\epsilon}-\varphi\left(\bar{z}_{\epsilon}\right)\right|^{n}} .
\end{aligned}
$$

To deal with $I_{\epsilon, 3}$, we first note that for any $y \in D_{\epsilon, 3}$, we have that

$$
\frac{1}{2}\left|y_{\epsilon}-\varphi\left(\bar{z}_{\epsilon}\right)\right| \leq\left|y-\varphi\left(\bar{z}_{\epsilon}\right)\right| \leq \frac{3}{2}\left|y_{\epsilon}-\varphi\left(\bar{z}_{\epsilon}\right)\right| .
$$

With inequality (101) (with $\theta=1$ ) on the Green's function, we then get that

$$
I_{\epsilon, 3} \leq C \frac{d\left(y_{\epsilon}, \partial \Omega\right) \mu_{\epsilon}^{\left(\frac{n}{2}-(n-1) \nu\right)\left(2^{\star}-1-p_{\epsilon}\right)}}{\left|y_{\epsilon}-\varphi\left(\bar{z}_{\epsilon}\right)\right|^{n(1-\nu)\left(2^{\star}-1-p_{\epsilon}\right)}} \int_{D_{\epsilon, 3}} \frac{d y}{\left|y_{\epsilon}-y\right|^{n-1}|\pi(y)|^{s-(1-\nu)\left(2^{\star}-1-p_{\epsilon}\right)}} .
$$

We let

$$
\theta_{\epsilon}=\frac{y_{\epsilon}-\varphi\left(\bar{z}_{\epsilon}\right)}{\left|y_{\epsilon}-\varphi\left(\bar{z}_{\epsilon}\right)\right|}+\frac{\left(\varphi_{0}\left(0, \bar{z}_{\epsilon}\right), 0,0\right)}{\left|y_{\epsilon}-\varphi\left(\bar{z}_{\epsilon}\right)\right|} .
$$

With (31), (40) and (69), we get that there exists $\theta_{0} \in \mathbb{R}^{n}$ such that $\left|\theta_{0}\right|=1$ and $\lim _{\epsilon \rightarrow 0} \theta_{\epsilon}=\theta_{0}$. With the change of variables $y=y_{\epsilon}+\left|y_{\epsilon}-\varphi\left(\bar{z}_{\epsilon}\right)\right| z$ and using (69), we get that

(75) $I_{\epsilon, 3} \leq$

$$
\begin{aligned}
& C \frac{d\left(y_{\epsilon}, \partial \Omega\right) \mu_{\epsilon}^{\left(\frac{n}{2}-(n-1) \nu\right)\left(2^{\star}-1-p_{\epsilon}\right)}}{\left|y_{\epsilon}-\varphi\left(\bar{z}_{\epsilon}\right)\right|^{(n-1)(1-\nu)\left(2^{\star}-1-p_{\epsilon}\right)+s-1}} \int_{|z|<\frac{1}{2}} \frac{d z}{|z|^{n-1}\left|\pi\left(\theta_{\epsilon}+z\right)\right|^{s-(1-\nu)\left(2^{\star}-1-p_{\epsilon}\right)}} \\
\leq & C \frac{d\left(y_{\epsilon}, \partial \Omega\right) \mu_{\epsilon}^{\left(\frac{n}{2}-(n-1) \nu\right)\left(2^{\star}-1-p_{\epsilon}\right)}}{\left|y_{\epsilon}-\varphi\left(\bar{z}_{\epsilon}\right)\right|^{(n-1)(1-\nu)\left(2^{\star}-1-p_{\epsilon}\right)+s-1}} \\
(76) & =o\left(\frac{d\left(y_{\epsilon}, \partial \Omega\right) \mu_{\epsilon}^{\frac{n}{2}}}{\left|y_{\epsilon}-\varphi\left(\bar{z}_{\epsilon}\right)\right|^{n}}\right)
\end{aligned}
$$

when $\epsilon \rightarrow 0$. Plugging (71), (73) and (76) in (70) and again using (69), we get that

$$
u_{\epsilon}\left(y_{\epsilon}\right) \leq C d\left(y_{\epsilon}, \partial \Omega\right)+C \frac{\mu_{\epsilon}^{\frac{n}{2}} d\left(y_{\epsilon}, \partial \Omega\right)}{\left(\mu_{\epsilon}^{2}+\left|y_{\epsilon}-\varphi\left(\bar{z}_{\epsilon}\right)\right|^{2}\right)^{\frac{n}{2}}}
$$

when $\epsilon \rightarrow 0$. This ends the proof of (68). 
Step 4.5. We claim that there exists $C>0$ such that

$$
\left|\nabla u_{\epsilon}(x)\right| \leq C+C \frac{\mu_{\epsilon}^{\frac{n}{2}}}{\left(\mu_{\epsilon}^{2}+\left|x-\varphi\left(\bar{z}_{\epsilon}\right)\right|^{2}\right)^{\frac{n}{2}}}
$$

for all $x \in \Omega$.

To prove the claim, as in Step 44, we just need to consider $\left(y_{\epsilon}\right)_{\epsilon>0} \in \Omega$ as in (69). We use Green's representation formula to write

$$
\nabla u_{\epsilon}\left(y_{\epsilon}\right)=\int_{\Omega} \nabla_{x} G_{\epsilon}\left(y_{\epsilon}, y\right) \frac{u_{\epsilon}(y)^{2^{\star}-1-p_{\epsilon}}}{|\pi(y)|^{s}} d y .
$$

With (68), we get that

$$
\left|\nabla u_{\epsilon}\left(y_{\epsilon}\right)\right| \leq J_{\epsilon, 1}+J_{\epsilon, 2}+J_{\epsilon, 3}
$$

where

$$
\begin{gathered}
J_{\epsilon, 1}:=C \int_{\Omega}\left|\nabla_{x} G_{\epsilon}\left(y_{\epsilon}, y\right)\right| \frac{d(y, \partial \Omega)^{2^{\star}-1-p_{\epsilon}}}{|\pi(y)|^{s}} d y \\
J_{\epsilon, 2}:=C \int_{\left|y_{\epsilon}-y\right|>\frac{1}{2}\left|y_{\epsilon}-\varphi\left(\bar{z}_{\epsilon}\right)\right|}\left|\nabla_{x} G_{\epsilon}\left(y_{\epsilon}, y\right)\right| \frac{\mu_{\epsilon}^{\frac{n}{2}\left(2^{\star}-1-p_{\epsilon}\right)} d(y, \partial \Omega)^{2^{\star}-1-p_{\epsilon}}}{|\pi(y)|^{s}\left(\mu_{\epsilon}^{2}+\left|y-\varphi\left(\bar{z}_{\epsilon}\right)\right|^{2}\right)^{\frac{n}{2}\left(2^{\star}-1-p_{\epsilon}\right)}} d y
\end{gathered}
$$

and

$$
J_{\epsilon, 3}:=C \int_{\left|y_{\epsilon}-y\right|<\frac{1}{2}\left|y_{\epsilon}-\varphi\left(\bar{z}_{\epsilon}\right)\right|}\left|\nabla_{x} G_{\epsilon}\left(y_{\epsilon}, y\right)\right| \frac{\mu_{\epsilon}^{\frac{n}{2}\left(2^{\star}-1-p_{\epsilon}\right)} d(y, \partial \Omega)^{2^{\star}-1-p_{\epsilon}}}{|\pi(y)|^{s}\left(\mu_{\epsilon}^{2}+\left|y-\varphi\left(\bar{z}_{\epsilon}\right)\right|^{2}\right)^{\frac{n}{2}\left(2^{\star}-1-p_{\epsilon}\right)}} d y .
$$

To estimate $J_{\epsilon, 1}$, use that the Green's function satisfies

$$
\left|\nabla_{x} G\left(y_{\epsilon}, y\right)\right| \leq \frac{C}{\left|y_{\epsilon}-y\right|^{n-1}}
$$

for all $y \in \Omega \backslash\left\{y_{\epsilon}\right\}$ and all $\epsilon>0$. We refer to [13 for the proof of this inequality. With (34), we then get that

$$
J_{\epsilon, 1} \leq C \int_{\Omega} \frac{d y}{\left|y_{\epsilon}-y\right|^{n-1}|\pi(y)|^{s-\left(2^{\star}-1-p_{\epsilon}\right)}} \leq C .
$$

For $J_{\epsilon, 2}$, we use that (see [13])

$$
\left|\nabla_{x} G\left(y_{\epsilon}, y\right)\right| \leq \frac{C d(y, \partial \Omega)}{\left|y_{\epsilon}-y\right|^{n}}
$$

for all $y \in \Omega \backslash\left\{y_{\epsilon}\right\}$ and all $\epsilon>0$. Plugging this inequality in $J_{\epsilon, 2}$ and performing computations similar to what was done in the proof of (73), we get that

$$
J_{\epsilon, 2} \leq C \frac{\mu_{\epsilon}^{\frac{n}{2}}}{\left(\mu_{\epsilon}^{2}+\left|x-\varphi\left(\bar{z}_{\epsilon}\right)\right|^{2}\right)^{\frac{n}{2}}} .
$$

Finally, to deal with $J_{\epsilon, 3}$, we again use estimate (79) on the Green's function combined with the same techniques as in the proof of (76) to obtain

$$
J_{\epsilon, 3} \leq C \frac{\mu_{\epsilon}^{\frac{n}{2}}}{\left(\mu_{\epsilon}^{2}+\left|x-\varphi\left(\bar{z}_{\epsilon}\right)\right|^{2}\right)^{\frac{n}{2}}} .
$$

Plugging (80), (81) and (82) in (78), we get (77) and Proposition 4.1. 


\section{Pohozaev identity And Proof of Theorem 1.1}

We first prove the following

Proposition 5.1. Let $\Omega$ be a smooth bounded domain of $\mathbb{R}^{n}, n \geq 3$, and let $\mathcal{P}$ be a linear vector subspace of $\mathbb{R}^{n}$ such that $2 \leq \operatorname{dim}_{\mathbb{R}} \mathcal{P} \leq n-1$. Assume that $s \in(0,2)$ and that (10) holds. For $\left(p_{\epsilon}\right)_{\epsilon>0} \in\left[0,2^{\star}-2\right)$ and $\left(a_{\epsilon}\right)_{\epsilon>0}$ as in (14), we consider $\left(u_{\epsilon}\right)_{\epsilon>0} \in H_{1,0}^{2}(\Omega) \cap C^{2}\left(\bar{\Omega} \backslash \mathcal{P}^{\perp}\right)$ such that (15), (16) and (17) hold. Then there exist $x_{0} \in \partial \Omega \cap \mathcal{P}^{\perp}, \gamma_{0} \geq 0$ and a family $\left(\mu_{\epsilon}\right)_{\epsilon>0} \in \mathbb{R}_{+}$such that $\lim _{\epsilon \rightarrow 0} \mu_{\epsilon}=0$ and

$$
\lim _{\epsilon \rightarrow 0} \frac{p_{\epsilon}}{\mu_{\epsilon}}=\frac{2(n-s)}{(n-2)^{2}} \mu_{s, \mathcal{P}}\left(\mathbb{R}_{-}^{n}\right)^{-\frac{n-s}{2-s}} \int_{\partial \mathbb{R}_{-}^{n}}\left(\frac{1}{2} I I_{x_{0}}(x, x)-\gamma_{0}\right)|\nabla v|^{2} d x,
$$

where $I I_{x_{0}}$ is the second fundamental form of $\partial \Omega$ at $x_{0}$.

Sections 5.1 to [5.3 below are devoted to the proof of Proposition [5.1] while Theorem 1.1 and Corollary 1.1 are proved in Step [5.4.

Step 5.1. We establish a Pohozaev-type identity for $u_{\epsilon}$. In the sequel, we let $\left(\bar{z}_{\epsilon}\right)_{\epsilon>0}$, $\left(\mu_{\epsilon}\right)_{\epsilon>0},\left(k_{\epsilon}\right)_{\epsilon>0}$ and $x_{0} \in \mathcal{P}^{\perp} \cap \partial \Omega$ as in Proposition 4.1. We also consider the chart $\varphi$ defined in (13). We let

$$
V_{\epsilon}=\Omega \cap \varphi\left(B_{\sqrt{\mu_{\epsilon}}}\left(\bar{z}_{\epsilon}\right)\right)=\varphi\left(\mathbb{R}_{-}^{n} \cap B_{\sqrt{\mu_{\epsilon}}}\left(\bar{z}_{\epsilon}\right)\right) .
$$

In particular,

$$
\partial V_{\epsilon}=\varphi\left(\mathbb{R}_{-}^{n} \cap \partial B_{\sqrt{\mu_{\epsilon}}}\left(\bar{z}_{\epsilon}\right)\right) \cup \varphi\left(B_{\sqrt{\mu_{\epsilon}}}\left(\bar{z}_{\epsilon}\right) \cap \partial \mathbb{R}_{-}^{n}\right)=V_{\epsilon}^{1} \cup V_{\epsilon}^{2} .
$$

In the sequel, we denote by $\nu(x)$ the outward normal vector at $x \in \partial V_{\epsilon}$ of the oriented hypersurface $\partial V_{\epsilon}$ (this is defined outside a null measure set). Let $\tilde{x}_{0} \in \mathbb{R}^{n}$. After integrations by parts (for instance, we refer to [12, 13]), we get that

$$
\begin{aligned}
& \left(\frac{n-2}{2}-\frac{n-s}{2^{\star}-p_{\epsilon}}\right) \int_{V_{\epsilon}} \frac{u_{\epsilon}^{2^{\star}-p_{\epsilon}}}{|\pi(x)|^{s}} d x-s \int_{V_{\epsilon}} \frac{\left(\tilde{x}_{0}, \pi(x)\right)}{|\pi(x)|^{s+2}} \cdot \frac{u_{\epsilon}^{2^{\star}-p_{\epsilon}}}{2^{\star}-p_{\epsilon}} d x \\
& +\int_{V_{\epsilon}}\left(a_{\epsilon}+\frac{\left(x-\tilde{x}_{0}\right)^{i} \partial_{i} a_{\epsilon}}{2}\right) u_{\epsilon}^{2} d x \\
& =\int_{\partial V_{\epsilon}}\left(-\frac{n-2}{2} u_{\epsilon} \partial_{\nu} u_{\epsilon}+\left(x-\tilde{x}_{0}, \nu\right) \frac{\left|\nabla u_{\epsilon}\right|^{2}}{2}-\left(x-\tilde{x}_{0}\right)^{i} \partial_{i} u_{\epsilon} \partial_{\nu} u_{\epsilon}\right. \\
& \left.-\frac{\left(x-\tilde{x}_{0}, \nu\right)}{2^{\star}-p_{\epsilon}} \cdot \frac{u_{\epsilon}^{2^{\star}}-p_{\epsilon}}{|\pi(x)|^{s}}+\frac{a_{\epsilon}\left(x-\tilde{x}_{0}, \nu\right)}{2} u_{\epsilon}^{2}\right) d \sigma
\end{aligned}
$$

for all $\epsilon>0$. Since $u_{\epsilon} \equiv 0$ on $\partial \Omega$, taking $\tilde{x}_{0}=\varphi\left(\bar{z}_{\epsilon}\right)$ in (84), we get that

$$
\begin{aligned}
& \left(\frac{n-2}{2}-\frac{n-s}{2^{\star}-p_{\epsilon}}\right) \int_{V_{\epsilon}} \frac{u_{\epsilon}^{2^{\star}-p_{\epsilon}}}{|\pi(x)|^{s}} d x-s \int_{V_{\epsilon}} \frac{\left(\varphi\left(\bar{z}_{\epsilon}\right), \pi(x)\right)}{|\pi(x)|^{s+2}} \cdot \frac{u_{\epsilon}^{2^{\star}-p_{\epsilon}}}{2^{\star}-p_{\epsilon}} d x \\
& +\int_{V_{\epsilon}}\left(a_{\epsilon}+\frac{\left(x-\varphi\left(\bar{z}_{\epsilon}\right)\right)^{i} \partial_{i} a_{\epsilon}}{2}\right) u_{\epsilon}^{2} d x \\
& =\int_{V_{\epsilon}^{1}}\left(-\frac{n-2}{2} u_{\epsilon} \partial_{\nu} u_{\epsilon}+\left(x-\varphi\left(\bar{z}_{\epsilon}\right), \nu\right) \frac{\left|\nabla u_{\epsilon}\right|^{2}}{2}\right. \\
& \left.-\left(x-\varphi\left(\bar{z}_{\epsilon}\right)\right)^{i} \partial_{i} u_{\epsilon} \partial_{\nu} u_{\epsilon}-\frac{\left(x-\varphi\left(\bar{z}_{\epsilon}\right), \nu\right)}{2^{\star}-p_{\epsilon}} \cdot \frac{u_{\epsilon}^{2^{\star}-p_{\epsilon}}}{|\pi(x)|^{s}}+\frac{a_{\epsilon}\left(x-\varphi\left(\bar{z}_{\epsilon}\right), \nu\right)}{2} u_{\epsilon}^{2}\right) d \sigma \\
& -\frac{1}{2} \int_{V_{\epsilon}^{2}}\left(x-\varphi\left(\bar{z}_{\epsilon}\right), \nu\right)\left|\nabla u_{\epsilon}\right|^{2} d \sigma .
\end{aligned}
$$


With (16), (41), (43), (44) and Proposition 3.3, we get that

$$
\begin{aligned}
& \left(\frac{(n-2)^{2}}{4(n-s)} \mu_{s, \mathcal{P}}\left(\mathbb{R}_{-}^{n}\right)^{\frac{2^{\star}}{2^{\star}-2}}+o(1)\right) p_{\epsilon}+s \int_{V_{\epsilon}} \frac{\left(\varphi\left(\bar{z}_{\epsilon}\right), \pi(x)\right)}{|\pi(x)|^{s+2}} \cdot \frac{u_{\epsilon}^{2^{\star}-p_{\epsilon}}}{2^{\star}-p_{\epsilon}} d x \\
& =\frac{1}{2} \int_{V_{\epsilon}^{2}}\left(x-\varphi\left(\bar{z}_{\epsilon}\right), \nu\right)\left|\nabla u_{\epsilon}\right|^{2} d \sigma+o\left(\mu_{\epsilon}\right) .
\end{aligned}
$$

Step 5.2. We deal with the RHS of (86). With a change of variable, we get that

$$
\begin{aligned}
& \int_{\varphi\left(B_{\sqrt{\mu_{\epsilon}}}\left(\bar{z}_{\epsilon}\right) \cap \partial \mathbb{R}_{-}^{n}\right)}\left(x-\varphi\left(\bar{z}_{\epsilon}\right), \nu\right)\left|\nabla u_{\epsilon}\right|^{2} d \sigma \\
& =(1+o(1)) \mu_{\epsilon} \int_{D_{\epsilon}}\left(\frac{\varphi\left(\bar{z}_{\epsilon}+k_{\epsilon} x\right)-\varphi\left(\bar{z}_{\epsilon}\right)}{k_{\epsilon}^{2}}, \nu \circ \varphi\left(\bar{z}_{\epsilon}+k_{\epsilon} x\right)\right)\left|\nabla v_{\epsilon}\right|_{\tilde{g}_{\epsilon}}^{2} \sqrt{\left|\tilde{g}_{\epsilon}\right|} d x,
\end{aligned}
$$

where the metric $\tilde{g}_{\epsilon}$ is such that $\left(\tilde{g}_{\epsilon}\right)_{i j}=\left(\partial_{i} \varphi, \partial_{j} \varphi\right)\left(\bar{z}_{\epsilon}+k_{\epsilon} x\right)$ for all $i, j=2, \ldots, n$, $v_{\epsilon}$ is as in Proposition 3.3 and

$$
D_{\epsilon}=B_{\frac{\sqrt{\mu_{\epsilon}}}{k_{\epsilon}}}(0) \cap\left\{x_{1}=0\right\} .
$$

Using the expression of $\varphi$ (see (13)), we get (see [12, 13] for details) that

$$
\begin{aligned}
& \left(\frac{\varphi\left(\bar{z}_{\epsilon}+k_{\epsilon} x\right)-\varphi\left(\bar{z}_{\epsilon}\right)}{k_{\epsilon}^{2}}, \nu \circ \varphi\left(\bar{z}_{\epsilon}+k_{\epsilon} x\right)\right) \\
& =\frac{1+o(1)}{k_{\epsilon}^{2}}\left(\varphi_{0}\left(\bar{z}_{\epsilon}+k_{\epsilon} x\right)-\varphi_{0}\left(\bar{z}_{\epsilon}\right)-k_{\epsilon} \sum_{i=2}^{n} x^{i} \partial_{i} \varphi\left(\bar{z}_{\epsilon}+k_{\epsilon} x\right)\right) \\
& =-\frac{1}{2} \sum_{i, j=2}^{n} \partial_{i j} \varphi\left(\bar{z}_{\epsilon}\right) x^{i} x^{j}+o_{\epsilon}(1)|x|^{2}
\end{aligned}
$$

for $\epsilon>0$ and $x \in D_{\epsilon}$. In this expression, $\lim _{\epsilon \rightarrow 0} o_{\epsilon}(1)=0$ uniformly in $D_{\epsilon}$. Plugging (88) into (87), using the estimate (44), Lebesgue's convergence theorem and (19), we get that

$$
\lim _{\epsilon \rightarrow 0} \frac{1}{\mu_{\epsilon}} \int_{\varphi\left(B_{\sqrt{\mu_{\epsilon}}}\left(\bar{z}_{\epsilon}\right) \cap \partial \mathbb{R}_{-}^{n}\right)}\left(x-\varphi\left(\bar{z}_{\epsilon}\right), \nu\right)\left|\nabla u_{\epsilon}\right|^{2} d \sigma=-\frac{1}{2} \int_{\partial \mathbb{R}_{-}^{n}} \partial_{i j} \varphi_{0}(0) x^{i} x^{j}|\nabla v|^{2} d x .
$$

Step 5.3. We deal with the second term of the LHS of (86). With the pointwise estimate (43) and a change of variables, we get that

$$
\begin{aligned}
& \int_{V_{\epsilon}} \frac{\left(\varphi\left(\bar{z}_{\epsilon}\right), \pi(x)\right)}{|\pi(x)|^{s+2}} \cdot \frac{u_{\epsilon}^{2^{\star}-p_{\epsilon}}}{2^{\star}-p_{\epsilon}} d x \\
& =\frac{1+o(1)}{\mu_{\epsilon}^{2}} \int_{D_{\epsilon}^{\prime}} \frac{\left(\pi \circ \varphi\left(\bar{z}_{\epsilon}\right), \pi \circ \varphi\left(\bar{z}_{\epsilon}+k_{\epsilon} x\right)\right)}{\left|\frac{\pi \circ \varphi\left(\bar{z}_{\epsilon}+k_{\epsilon} x\right)}{k_{\epsilon}}\right|^{s+2}} \cdot \frac{v_{\epsilon}^{2^{\star}-p_{\epsilon}}}{2^{\star}-p_{\epsilon}} d x
\end{aligned}
$$

when $\epsilon \rightarrow 0$, where

$$
D_{\epsilon}^{\prime}:=B_{R \frac{\sqrt{\mu_{\epsilon}}}{k_{\epsilon}}}(0) \cap\left\{x_{1}<0\right\} .
$$


With the explicit expression of $\varphi$ (see (13)) and noting $x=\left(x_{1}, y, z\right)$ as in (13), we get that

$$
\begin{aligned}
& \int_{V_{\epsilon}} \frac{\left(\varphi\left(\bar{z}_{\epsilon}\right), \pi(x)\right)}{|\pi(x)|^{s+2}} \cdot \frac{u_{\epsilon}^{2^{\star}-p_{\epsilon}}}{2^{\star}-p_{\epsilon}} d x \\
& =(1+o(1)) \frac{\varphi_{0}\left(0, z_{\epsilon}\right)}{k_{\epsilon}} \int_{D_{\epsilon}^{\prime}} \frac{x_{1}+\frac{\varphi_{0}\left(k_{\epsilon} y, z_{\epsilon}+k_{\epsilon} z\right)}{k_{\epsilon}}}{\left|\pi\left(x_{1}+\frac{\varphi_{0}\left(k_{\epsilon} y, z_{\epsilon}+k_{\epsilon} z\right)}{k_{\epsilon}}, y, z\right)\right|^{s+2}} \frac{v_{\epsilon}^{2^{\star}-p_{\epsilon}}}{2^{\star}-p_{\epsilon}} d x
\end{aligned}
$$

when $\epsilon \rightarrow 0$. With point (iii) of (13), the estimate (43) and Lebesgue's convergence theorem, we get that

$$
\begin{aligned}
& \int_{V_{\epsilon}} \frac{\left(\varphi\left(\bar{z}_{\epsilon}\right), \pi(x)\right)}{|\pi(x)|^{s+2}} \frac{u_{\epsilon}^{2^{\star}-p_{\epsilon}}}{2^{\star}-p_{\epsilon}} d x \\
& =\frac{\varphi_{0}\left(0, z_{\epsilon}\right)}{\mu_{\epsilon}}\left(\int_{\mathbb{R}_{-}^{n}} \frac{x_{1} v^{2^{\star}}}{2^{\star}|\pi(x)|^{s+2}} d x+o(1)\right)
\end{aligned}
$$

where $\lim _{\epsilon \rightarrow 0} o(1)=0$. Plugging (89) and (90) into (86) and noting that $\varphi_{0}\left(0, z_{\epsilon}\right) \leq$ 0 (see (33)), we get that

$$
\begin{aligned}
& \left(\frac{(n-2)^{2}}{4(n-s)} \mu_{s, \mathcal{P}}\left(\mathbb{R}_{-}^{n}\right)^{\frac{n-s}{2-s}}+o(1)\right) p_{\epsilon}+\left(\int_{\mathbb{R}_{-}^{n}} \frac{s\left|x_{1}\right| v^{2^{\star}}}{2^{\star}|\pi(x)|^{s+2}} d x+o(1)\right) \frac{\left|\varphi_{0}\left(0, z_{\epsilon}\right)\right|}{\mu_{\epsilon}} \\
(91)= & \left(-\frac{1}{4} \int_{\partial \mathbb{R}_{-}^{n}} \partial_{i j} \varphi_{0}(0) x^{i} x^{j}|\nabla v|^{2} d x+o(1)\right) \cdot \mu_{\epsilon}
\end{aligned}
$$

where $\lim _{\epsilon \rightarrow 0} o(1)=0$. In particular, we get that $\left|\varphi_{0}\left(0, z_{\epsilon}\right)\right|=O\left(\mu_{\epsilon}^{2}\right)$ when $\epsilon \rightarrow 0$. We let

$$
\gamma_{0}:=-\lim _{\epsilon \rightarrow 0} \frac{\varphi_{0}\left(0, z_{\epsilon}\right)}{\mu_{\epsilon}^{2}} \geq 0 .
$$

With (91), we get that

$$
\begin{aligned}
& \lim _{\epsilon \rightarrow 0} \frac{(n-2)^{2}}{4(n-s)} \mu_{s, \mathcal{P}}\left(\mathbb{R}_{-}^{n}\right)^{\frac{n-s}{2-s}} \frac{p_{\epsilon}}{\mu_{\epsilon}} \\
& =-\frac{1}{4} \int_{\partial \mathbb{R}_{-}^{n}} \partial_{i j} \varphi_{0}(0) x^{i} x^{j}|\nabla v|^{2} d x-\gamma_{0} \frac{s}{2^{\star}} \int_{\mathbb{R}_{-}^{n}} \frac{\left|x_{1}\right| \cdot v^{2^{\star}}}{|\pi(x)|^{s+2}} d x .
\end{aligned}
$$

Taking $\tilde{x_{0}}=\vec{e}_{1}$ in (84), using a change of variable and the arguments used to prove (90), we get that

$$
\frac{s}{2^{\star}} \int_{\mathbb{R}_{-}^{n}} \frac{\left|x_{1}\right| v^{2^{\star}}}{|\pi(x)|^{s+2}} d x=\frac{1}{2} \int_{\partial \mathbb{R}_{-}^{n}}|\nabla v|^{2} d x .
$$

We consider the second fundamental form associated to $\partial \Omega$, namely

$$
I I_{p}(x, y)=\left(d \nu_{p} x, y\right)
$$

for all $p \in \partial \Omega$ and all $x, y \in T_{x_{0}} \partial \Omega$ (recall that $\nu$ is the outward normal vector at the hypersurface $\partial \Omega)$. In the basis $\left(\vec{e}_{1}, \ldots, \vec{e}_{n}\right)$, the matrix of the bilinear form $I I_{x_{0}}$ is $-D_{0}^{2} \varphi_{0}$, where $D_{0}^{2} \varphi_{0}$ is the Hessian matrix of $\varphi_{0}$ at 0 . With this remark, (91) 
and (92), we get that

$$
\lim _{\epsilon \rightarrow 0} \frac{p_{\epsilon}}{\mu_{\epsilon}}=\frac{2(n-s)}{(n-2)^{2}} \mu_{s, \mathcal{P}}\left(\mathbb{R}_{-}^{n}\right)^{-\frac{n-s}{2-s}} \int_{\partial \mathbb{R}_{-}^{n}}\left(\frac{1}{2} I I_{x_{0}}(x, x)-\gamma_{0}\right)|\nabla v|^{2} d x,
$$

where $\gamma_{0} \geq 0$. This ends the proof of Proposition 5.1

Step 5.4. We are now in a position to prove Theorem 1.1. Points (A) and (B) of Theorem 1.1 are direct consequences of Propositions 2.1 and 2.2. To establish Part (C) of Theorem 1.1] assume that (10) holds and let us suppose that there are no extremals for (6). It follows from Proposition 3.2 that there exists $\left(u_{\epsilon}\right)_{\epsilon>0} \in H_{1,0}^{2}(\Omega)$ such that (15) and (16) hold with $p_{\epsilon}=\epsilon$ and $a_{\epsilon} \equiv 0$. Since there are no extremals, it follows from Proposition 3.2 that (17) holds. We apply Proposition 5.1 and we get that

$$
\lim _{\epsilon \rightarrow 0} \frac{\epsilon}{\mu_{\epsilon}}=\frac{2(n-s)}{(n-2)^{2}} \mu_{s, \mathcal{P}}\left(\mathbb{R}_{-}^{n}\right)^{-\frac{n-s}{2-s}} \int_{\partial \mathbb{R}_{-}^{n}}\left(\frac{1}{2} I I_{x_{0}}(x, x)-\gamma_{0}\right)|\nabla v|^{2} d x,
$$

where $x_{0} \in \mathcal{P}^{\perp} \cap \partial \Omega$ and $\gamma_{0} \geq 0$. We then get that

$$
\int_{\partial \mathbb{R}_{-}^{n}} I I_{x_{0}}(x, x)|\nabla v|^{2} d x \geq 0 .
$$

Assume that we are in the first case of point $(\mathrm{C})$ of Theorem 1.1. We then get that $I I_{x_{0}}(x, x) \leq 0$ for all $x \in \partial \mathbb{R}_{-}^{n}$, but $I I_{x_{0}}(x, x) \not \equiv 0$, contradicting (93).

To relate our main result to conditions on the mean curvature, we now assume that $\mathcal{P} \cap T_{x} \partial \Omega$ and $\mathcal{P}^{\perp}$ are orthogonal with respect to the bilinear form $I I_{x_{0}}$. We get in the coordinates (12) and the chart (13) that $\left(I I_{x_{0}}\right)_{i j}=0$ when $i \in\{2, \ldots, k\}$ and $j \in\{k+1, n\}$. In particular, we have with (93) that

$$
\left(\sum_{i, j=2}^{k}\left(I I_{x_{0}}\right)_{i j} \int_{\partial \mathbb{R}_{-}^{n}} x^{i} x^{j}|\nabla v|^{2} d x\right)+\left(\sum_{i, j=k+1}^{n}\left(I I_{x_{0}}\right)_{i j} \int_{\partial \mathbb{R}_{-}^{n}} x^{i} x^{j}|\nabla v|^{2} d x\right) \geq 0
$$

The matrix of the second fundamental form of $\partial \Omega \cap\left(\mathcal{P}^{\perp}+\left(T_{x_{0}} \partial \Omega\right)^{\perp}\right)$ at $x_{0}$ with respect to a given vector $\vec{X}$ is $\left(\left(I I_{x_{0}}(\vec{X})\right)_{i j}\right)_{i, j \geq k+1}=-\left(\partial_{i j} \varphi_{0}(0) X^{1}\right)_{i, j \geq k+1}$. Since $\nabla \varphi_{0}(0)=0$ and $\varphi_{0}(0, z) \leq 0$ for $z$ close to 0 , we get that for any direction $\vec{X}$, the principal curvatures of $\partial \Omega \cap\left(x_{0}+\left(\mathcal{P}^{\perp}+\left(T_{x_{0}} \partial \Omega\right)^{\perp}\right)\right)$ at $x_{0}$ have a sign. If the mean curvature vector of $\partial \Omega \cap\left(x_{0}+\left(\mathcal{P}^{\perp}+\left(T_{x_{0}} \partial \Omega\right)^{\perp}\right)\right)$ at $x_{0}$ is assumed to be null, it then follows that the second fundamental form of $\partial \Omega \cap\left(\mathcal{P}^{\perp}+\left(T_{x_{0}} \partial \Omega\right)^{\perp}\right)$ at $x_{0}$ is null, and we then get from (94) that

$$
\sum_{i, j=2}^{k}\left(I I_{x_{0}}\right)_{i j} \int_{\partial \mathbb{R}_{-}^{n}} x^{i} x^{j}|\nabla v|^{2} d x \geq 0
$$

Here, $v \in H_{1,0}^{2}\left(\mathbb{R}_{-}^{n}\right)$ is positive, it verifies that $\Delta v=\frac{v^{2^{\star}-1}}{|\pi(x)|^{s}}$ weakly, and $v(x) \leq$ $C\left(1+|x|^{2}\right)^{-n}$ for all $x \in \mathbb{R}_{-}^{n}$ (this last statement is a consequence of (19) and (43)). It follows from Proposition[1.1 that there exists $\tilde{v}$ such that $v\left(x_{1}, y, z\right)=\tilde{v}\left(x_{1},|y|, z\right)$. With this symmetry property, with (95) we get that $\sum_{i=1}^{k}\left(I I_{x_{0}}\right)_{i i} \geq 0$, and then the mean curvature at $x_{0}$ of $\partial \Omega$ is non-negative. This contradicts assumption (2) of case (C) of Theorem 1.1. This ends the proof of the theorem. 
Concerning Corollary 1.1, the subcritical problem yields families of positive solutions to (15) and (16) with $a_{\epsilon} \equiv a$ and $p_{\epsilon}=\epsilon$. The proof of Corollary 1.1 then proceeds as in the proof of Theorem 1.1 .

\section{Proof of Proposition 1.2}

We let $\Omega$ and $\mathcal{P}$ be as in Proposition 1.2. In particular $\operatorname{dim}_{\mathbb{R}} \mathcal{P}=1$. The proof of case (B) of Proposition 1.2 proceeds exactly as in the proof of Proposition 2.2 Concerning case $(\mathrm{C})$, we claim that $\mu_{s, \mathcal{P}}(\Omega)=0$ when $s \in[1,2)$. Indeed, taking $u \in C_{c}^{\infty}(\Omega)$ such that $u\left(x_{0}\right)=1$, where $x_{0} \in \mathcal{P}^{\perp} \cap \Omega$, it is easily checked that $\int_{\Omega} \frac{u^{2^{\star}}}{|\pi(x)|^{s}} d x=+\infty$, and then $\mu_{s, \mathcal{P}}(\Omega)=0$ is not achieved. When $s \in(0,1)$ in case (A), the proof of nonachievement proceeds as in the proof of Proposition 2.1

We are left with case (C) of Proposition 1.2, that is, $\mathcal{P}^{\perp} \cap \Omega=\emptyset$ and $\mathcal{P}^{\perp} \cap \partial \Omega \neq \emptyset$. Up to a change of coordinates, we assume that $\mathcal{P}^{\perp}=\left\{x_{1}=0\right\}, \Omega \subset \mathbb{R}_{-}^{n}$ and $0 \in \mathcal{P}^{\perp} \cap \partial \Omega$ and $|\pi(x)|=\left|x_{1}\right|$. In particular, it follows from the Sobolev inequality and the Hardy inequality that

$$
\mu_{s, \mathcal{P}}\left(\mathbb{R}_{-}^{n}\right):=\inf \left\{\frac{\int_{\mathbb{R}_{-}^{n}}|\nabla u|^{2} d x}{\left(\int_{\mathbb{R}_{-}^{n}} \frac{|u|^{\star}}{\left|x_{1}\right|^{s}} d x\right)^{\frac{2}{2 \star}}} \mid u \in H_{1,0}^{2}\left(\mathbb{R}_{-}^{n}\right) \backslash\{0\}\right\}>0 .
$$

Since $\Omega \subset \mathbb{R}_{-}^{n}$, we get that $\mu_{s, \mathcal{P}}(\Omega) \geq \mu_{s, \mathcal{P}}\left(\mathbb{R}_{-}^{n}\right)$. With arguments similar to the proof of Proposition 3.1 we also get the reverse inequality, and then $\mu_{s, \mathcal{P}}(\Omega)=$ $\mu_{s, \mathcal{P}}\left(\mathbb{R}_{-}^{n}\right)$. In particular, an extremal for $\mu_{s, \mathcal{P}}(\Omega)$ is an extremal for $\mu_{s, \mathcal{P}}\left(\mathbb{R}_{-}^{n}\right)$ and vice versa. As in the proof of Proposition 2.1 the maximum principle yields a contradiction.

\section{Appendix: Regularity of Weak solutions}

In this Appendix, we prove the following regularity result:

Proposition 7.1. Let $\Omega$ be a smooth bounded domain of $\mathbb{R}^{n}, n \geq 3$. Let $\mathcal{P} \subset \mathbb{R}^{n}$ be a $k$-dimensional linear subspace of $\mathbb{R}^{n}$, where $2 \leq k \leq n-1$. We assume that

$$
\mathcal{P}^{\perp} \cap \Omega=\emptyset \text { and } \mathcal{P}^{\perp} \cap \partial \Omega \neq \emptyset .
$$

We let $s \in(0,2)$ and $a \in C^{0, \alpha}(\bar{\Omega})$, where $\alpha \in(0,1)$. We let $\epsilon \in\left[0,2^{\star}-2\right)$ and consider $u \in H_{1,0}^{2}(\Omega)$ a weak solution of

$$
\Delta u+a u=\frac{|u|^{2^{\star}-2-\epsilon} u}{|\pi(x)|^{s}} \text { in } \mathcal{D}^{\prime}(\Omega) .
$$

Then $u \in C^{1}(\bar{\Omega}) \cap C^{2, \alpha}\left(\bar{\Omega} \backslash \mathcal{P}^{\perp}\right)$.

Proof of Proposition 7.1. Note that since $2^{\star}<\frac{2 n}{n-2}$, it follows from standard elliptic theory that $u \in C^{2, \alpha}\left(\bar{\Omega} \backslash \mathcal{P}^{\perp}\right)$. In particular, $u \in C^{2, \alpha}(\Omega)$.

Step 7.1. We claim that

$$
u \in L^{p}(\Omega)
$$

for all $p \geq 1$. Indeed, the proof is similar to the case $\mathcal{P}=\mathbb{R}^{n}$ provided in [12, 13]. We omit the proof and refer to [12, 13, for the details.

In particular, we get that $\frac{|u|^{2^{\star}-2-\epsilon} u}{|\pi(x)|^{s}} \in L^{p}(\Omega)$ for all $1 \leq p<\frac{k}{s}$. In the case $k=n$, we take $p>\frac{n}{2}$, and then $u \in L^{\infty}(\Omega)$. A bootstrap argument (see also [10]) 
then yields that $u \in C^{1}(\bar{\Omega})$. However, in the general case $2 \leq k \leq n-1$, such an argument using standard elliptic theory does not hold, and we have to use the Green's function to prove the proposition.

Step 7.2. We let $\theta \in(0, \min \{2-s, 1\})$. We claim that there exists $C>0$ such that

$$
|u(x)| \leq C d(x, \partial \Omega)^{\theta}
$$

for a.e. $x \in \Omega$.

Proof of the claim. We let $\left(\eta_{k}\right)_{k \in \mathbb{N}} \in C_{c}^{\infty}(\Omega)$ such that $0 \leq \eta_{k} \leq 1$ for all $k$ and $\eta_{k}(x)=1$ for $d(x, \partial \Omega) \geq 2 k^{-1}$. We let $\left(u_{k}\right)_{k \in \mathbb{N}} \in H_{1,0}^{2}(\Omega)$ such that

$$
\Delta u_{k}=\eta_{k}\left(\frac{|u|^{2^{\star}-2-\epsilon} u}{|\pi(x)|^{s}}-a u\right) .
$$

Since $u \in C^{2}(\Omega)$ and $\Omega \cap \mathcal{P}^{\perp}=\emptyset$, we get that $u_{k} \in C^{2}(\bar{\Omega})$ for all $k \in \mathbb{N}$. We let $G$ be the Green's function for $\Delta$ with Dirichlet boundary condition. It follows from Green's representation formula that

$$
u_{k}(x)=\int_{\Omega} G(x, y) \eta_{k}(y)\left(\frac{|u|^{2^{\star}-1-\epsilon}(y)}{|\pi(y)|^{s}}-a u\right) d y
$$

for a.e. $x \in \Omega$. It follows from Theorem 9.1 of [13] that there exists $C>0$ such that

$$
0<G(x, y) \leq C \frac{d(x, \partial \Omega)^{\theta}}{|x-y|^{n-2+\theta}}
$$

for all $x, y \in \Omega, x \neq y$. Plugging this inequality in (100) and using Hölder's inequality, we get that

$$
\begin{aligned}
\left|u_{k}(x)\right| \leq & C d(x, \partial \Omega)^{\theta} \int_{\Omega} \frac{1}{|x-y|^{n-2+\theta}}\left(\frac{|u(y)|^{2^{\star}-1-\epsilon}}{|\pi(y)|^{s}}+|u(y)|\right) d y \\
\leq & C d(x, \partial \Omega)^{\theta}\left\||u|^{2^{\star}-1-\epsilon}\right\|_{q}\left(\int_{\Omega} \frac{d y}{|x-y|^{p(n-2+\theta)}|\pi(y)|^{s p}}\right)^{\frac{1}{p}} \\
& +C d(x, \partial \Omega)^{\theta}\|u\|_{q^{\prime}}\left(\int_{\Omega} \frac{d y}{|x-y|^{p^{\prime}(n-2+\theta)}}\right)^{\frac{1}{p^{\prime}}}
\end{aligned}
$$

where $p, q, p^{\prime}, q^{\prime}>1$ are such that $\frac{1}{p}+\frac{1}{q}=\frac{1}{p^{\prime}}+\frac{1}{q^{\prime}}=1$. Since $\theta \in(0,1)$ and (97) holds, we get that there exists $C>0$ such that for $p, p^{\prime}>1$ sufficiently close to 1 , we have that

$$
|u(x)| \leq C d(x, \partial \Omega)^{\theta}\left(\int_{\Omega} \frac{d y}{|x-y|^{p(n-2+\theta)}|\pi(y)|^{s p}}\right)^{\frac{1}{p}}+C d(x, \partial \Omega)^{\theta}
$$

for all $x \in \Omega$. For simplicity, up to a change of coordinates, we write any $y \in \mathbb{R}^{n}$ as $y=\left(y^{\prime}, y^{\prime \prime}\right)$, where $y^{\prime}=\pi(y) \in \mathbb{R}^{k}=\mathcal{P}$ and $y^{\prime \prime} \in \mathbb{R}^{n-k}=\mathcal{P}^{\perp}$. We let $R>0$ such that $\Omega \subset B_{R}^{k}(0) \times B_{R}^{n-k}(0)$ (the product of the ball of radius $R$ in $\mathbb{R}^{k}$ and the ball 
of radius $R$ in $\mathbb{R}^{n-k}$ ). We then get with a change of variable that

$$
\begin{aligned}
& \int_{\Omega} \frac{d y}{|x-y|^{p(n-2+\theta)}|\pi(y)|^{s p}} \\
& \leq C \int_{B_{R}^{k}(0)} \frac{1}{\left|y^{\prime}\right|^{p s}} \int_{B_{R}(0)^{n-k}}\left(\frac{1}{\left|x^{\prime}-y^{\prime}\right|^{p(n-2+\theta)}+\left|x^{\prime \prime}-y^{\prime \prime}\right|^{p(n-2+\theta)}}\right) d y^{\prime} \\
& \leq C \int_{B_{R}^{k}(0)} \frac{d z^{\prime \prime}}{\left|y^{\prime}\right|^{p s}\left|x^{\prime}-y^{\prime}\right|^{p(n-2+\theta)+k-n}} \int_{B_{\frac{2 R}{\left|x^{\prime}-y^{\prime}\right|}}(0)^{n-k}}\left(\frac{d y^{\prime}}{1+\left|z^{\prime \prime}\right|^{p(n-2+\theta)}}\right) d y^{\prime} \\
& \leq C \int_{B_{R}^{k}(0)} \frac{d y^{\prime}}{\left|y^{\prime}\right|^{p s}\left|x^{\prime}-y^{\prime}\right|^{p(n-2+\theta)+k-n}} \leq C
\end{aligned}
$$

for all $\left(x^{\prime}, x^{\prime \prime}\right) \in \Omega$. Here, we have taken $p>1$ close to 1 and we have used the fact that $s \in(0,2)$. Plugging this inequality in (102), we get that there exists $C>0$ such that

$$
\left|u_{k}(x)\right| \leq C d(x, \partial \Omega)^{\theta}
$$

for all $x \in \Omega$ and all $k \in \mathbb{N}$. Multiplying (99) by $u_{k}$, integrating over $\Omega$, using the fact that $u \in H_{1,0}^{2}(\Omega)$, and by the inequalities (10) and (103), we get that there exists $C>0$ such that $\left\|u_{k}\right\|_{H_{1,0}^{2}(\Omega)} \leq C$ for all $k \in \mathbb{N}$. It then follows that there exists $\tilde{u} \in H_{1,0}^{2}(\Omega)$ such that $u_{k} \rightarrow \tilde{u}$ weakly in $H_{1,0}^{2}(\Omega)$ when $k \rightarrow+\infty$ and $\lim _{k \rightarrow+\infty} u_{k}(x)=\tilde{u}(x)$ for a.e. $x \in \Omega$. The function $\tilde{u}$ verifies $\Delta \tilde{u}=\frac{|u|^{2^{\star}-2-\epsilon}}{|\pi(x)|^{s}}-a u$ in $\mathcal{D}^{\prime}(\Omega)$. Since $\Delta$ is coercive, it then follows from (96) that $\tilde{u}=u$. With (103), we then get (98).

Step 7.3. We claim that there exists $C>0$ such that

$$
|u(x)| \leq C d(x, \partial \Omega)
$$

for a.e. $x \in \Omega$.

Proof of the claim. Indeed, we let $\theta_{0} \in(0,1)$ such that there exists $C>0$ such that $|u(x)| \leq C d(x, \partial \Omega)^{\theta_{0}}$. With (34), we get that there exists $C>0$ such that $|u(x)| \leq C|\pi(x)|^{\theta_{0}}$ for all $x \in \Omega$. We let $\theta \in(0,1)$. It follows from Green's representation formula and (101) that there exists $C>0$ such that

$$
\begin{aligned}
\left|u_{k}(x)\right| & =\left|\int_{\Omega} G(x, y) \eta_{k}(y)\left(\frac{|u|^{2^{\star}-1-\epsilon}(y)}{|\pi(y)|^{s}}-a u\right) d y\right| \\
& \leq C d(x, \partial \Omega)^{\theta}+C \int_{\Omega} \frac{d(x, \partial \Omega)^{\theta}}{|x-y|^{n-2+\theta}|\pi(y)|^{s-\theta_{0}\left(2^{\star}-1-\epsilon\right)}} d y .
\end{aligned}
$$

We proceed as in Step $\left[73\right.$ and get that $|u(x)| \leq C d(x, \partial \Omega)^{\theta}$ for some $\theta>\theta_{0}$. The claim follows by induction.

Step 7.4. We claim that $u \in C^{1}(\bar{\Omega})$.

Proof of the claim. With inequality (104) and the method used in Step 72, we get that

$$
\lim _{k \rightarrow+\infty} u_{k}(x)=\int_{\Omega} G(x, y)\left(\frac{|u|^{2^{\star}-2-\epsilon} u(y)}{|\pi(y)|^{s}}-a u\right) d y
$$


and

$$
\lim _{k \rightarrow+\infty} \nabla u_{k}(x)=\int_{\Omega} \nabla_{x} G(x, y)\left(\frac{|u|^{2^{\star}-2-\epsilon} u(y)}{|\pi(y)|^{s}}-a u\right) d y
$$

uniformly for $x \in \bar{\Omega}$. Since $u_{k} \rightarrow u$ in $H_{1,0}^{2}(\Omega)$ when $k \rightarrow+\infty$, we get that $u \in C^{1}(\bar{\Omega})$.

\section{REFERENCES}

[1] Badiale, M.; Tarantello, G. A Sobolev-Hardy inequality with applications to a nonlinear elliptic equation arising in astrophysics. Arch. Ration. Mech. Anal., 163 (2002), 259-293. MR.1918928 (2003j:35079)

[2] Atkinson, F.V.; Peletier, L.A. Elliptic equations with nearly critical growth. J. Differential Equations, 70 (1987), 349-365. MR 915493 (89e:35054)

[3] Brézis, H.; Peletier, L.A. Asymptotics for elliptic equations involving critical Sobolev exponent. Partial differential equations and the calculus of variations, Vol. I, 149-192. Progr. Nonlinear Differential Equations Appl., 1, Birkhäuser Boston, Boston, MA, 1989. MR.1034005 (91a:35030)

[4] Caffarelli, L.; Gidas, B.; Spruck, J. Asymptotic symmetry and local behavior of semilinear elliptic equations with critical Sobolev growth. Comm. Pure Appl. Math., 42 (1989), 271-297. MR982351 (90c:35075)

[5] Druet, O. The best constants problem in Sobolev inequalities. Math. Ann., 314 (1999), 327346. MR:1697448 (2000d:58033)

[6] Druet, O. Elliptic equations with critical Sobolev exponent in dimension 3. Ann. Inst. H. Poincaré Anal. Non Linéaire, 19 (2002), 125-142. MR.1902741 (2003f:35104)

[7] Druet, O.; Hebey, E. Elliptic equations of Yamabe type. IMRS Int. Math. Res. Surv., 2005, 1-113. MR2148873 (2006b:53046)

[8] Druet, O.; Hebey, E.; Robert, F. Blow up theory for elliptic PDE's in Riemannian geometry. Mathematical Notes, 45. Princeton University Press, Princeton, NJ, 2004. Announcement in "A $C^{0}$-theory for the blow up of second order elliptic equations of critical Sobolev growth". Electron. Res. Announc. Amer. Math. Soc., 9 (2003), 19-25. MR1988868 (2004c:58046)

[9] Caffarelli, L.; Kohn, R.; Nirenberg, L. First order interpolation inequality with weights, Compositio Math., 53 (1984), 259-275. MR768824 (86c:46028)

[10] Egnell, H., Positive solutions of semilinear equations in cones. Trans. Amer. Math. Soc., 330 (1992), 191-201. MR1034662 (92f:35018)

[11] Ghoussoub, N.; Kang, X.S. Hardy-Sobolev Critical Elliptic Equations with Boundary Singularities. Ann. Inst. H. Poincaré Anal. Non Linéaire, 21 (2004), 767-793. MR2097030 (2005i:35086)

[12] Ghoussoub, N.; Robert, F. The effect of curvature on the best constant in the Hardy-Sobolev inequalities. Geom. Funct. Anal., 16 (2006), no. 6, 1201-1245. MR2276538 (2007k:35085)

[13] Ghoussoub, N.; Robert, F. Concentration estimates for Emden-Fowler equations with boundary singularities and critical growth. IMRP Int. Math. Res. Pap., (2006), 21867, 1-85. MR2210661 (2006k:35094)

[14] Ghoussoub, N.; Yuan, C. Multiple solutions for quasi-linear PDEs involving the critical Sobolev and Hardy exponents. Trans. Amer. Math. Soc., 352 (2000), 5703-5743. MR1695021 (2001b:35109)

[15] Gidas, B.; Ni, W.M.; Nirenberg, L. Symmetry and related properties via the maximum principle. Comm. Math. Phys., 68 (1979), 209-243. MR544879 (80h:35043)

[16] Gilbarg, G.; Trudinger, N.S. Elliptic partial differential equations of second order. Second edition. Grundlehren der Mathematischen Wissenschaften, 224, Springer-Verlag, Berlin, 1983. MR737190 (86c:35035)

[17] Han, Z.C. Asymptotic approach to singular solutions for nonlinear elliptic equations involving critical Sobolev exponent. Ann. Inst. H.Poincaré. Anal. Non Linéaire, 8 (1991), 159-174. MR 1096602 (92c:35047)

[18] Hebey, E.; Vaugon, M. The best constant problem in the Sobolev embedding theorem for complete Riemannian manifolds. Duke Math. J., 79 (1995), 235-279. MR.1340298|(96c:53057)

[19] Hebey, E.; Vaugon, M. From best constants to critical functions. Math. Z., 237 (2001), 737-767. MR,1854089 (2002h:58061) 
[20] Maz'ya, V.G. Sobolev spaces. Springer Series in Soviet Mathematics, Springer-Verlag, Berlin, 1985. MR817985 (87g:46056)

[21] Robert, F. Asymptotic behaviour of a nonlinear elliptic equation with critical Sobolev exponent. The radial case II. NoDEA Nonlinear Differential Equations Appl., 9 (2002), 361-384. MR1917379 (2003i:35058)

[22] Robert, F. Critical functions and optimal Sobolev inequalities. Math. Z., 249 (2005), 485-492. MR2121735 (2007m:53035)

[23] Schoen, R.; Zhang, D. Prescribed scalar curvature on the $n$-sphere. Calc. Var. Partial Differential Equations, 4 (1996), 1-25. MR1379191 (97j:58027)

[24] Struwe, M. Variational methods. Applications to nonlinear partial differential equations and Hamiltonian systems. Third edition. Ergebnisse der Mathematik und ihrer Grenzgebiete, 34 . Springer-Verlag, Berlin, 2000. MR1736116 (2000i:49001)

Department of Mathematics, University of British Columbia, Vancouver, British Columbia, CANAda

E-mail address: nassif@math.ubc.ca

Laboratoire J.A. Dieudonné, Université de Nice Sophia-Antipolis, Parc Valrose, 06108 Nice CEDEX 2, France

E-mail address: frobert@math.unice.fr 